\title{
Direct numerical simulation of a transitional temporal mixing layer laden with multicomponent-fuel evaporating drops using continuous thermodynamics
}

\author{
P. C. Le Clercq \\ California Institute of Technology, Pasadena, California 91125 \\ J. Bellan ${ }^{\text {a) }}$ \\ Jet Propulsion Laboratory and California Institute of Technology, Pasadena, California 91109-8099
}

(Received 15 September 2003; accepted 29 January 2004; published online 28 April 2004)

\begin{abstract}
A model of a temporal three-dimensional mixing layer laden with fuel drops of a liquid containing a large number of species is derived. The fuel model is based on continuous thermodynamics, whereby the composition is statistically described through a distribution function parametrized on the species molar weight. The drop temperature is initially lower than that of the carrier gas, leading to drop heat up and evaporation. The model describing the changes in the multicomponent (MC) fuel drop composition and in the gas phase composition due to evaporation encompasses only two more conservation equations when compared with the equivalent single-component (SC) fuel formulation. Single drop results of a MC fuel having a sharply peaked distribution are shown to compare favorably with a validated SC-fuel drop simulation. Then, single drop comparisons are performed between results from MC fuel and a representative SC fuel used as a surrogate of the MC fuel. Further, two mixing layer simulations are conducted with a $\mathrm{MC}$ fuel and they are compared to representative SC-fuel simulations conducted elsewhere. Examination of the results shows that although the global layer characteristics are generally similar in the SC and MC situations, the MC layers display a higher momentum-thickness-based Reynolds number at transition. Vorticity analysis shows that the SC layers exhibit larger vortical activity than their MC counterpart. An examination of the drop organization at transition shows more structure and an increased drop-number density for MC simulations in regions of moderate and high strain. These results are primarily attributed to the slower evaporation of MC-fuel drops than of their SC counterpart. This slower evaporation is due to the lower volatility of the higher molar weight species, and also to condensation of already-evaporated species on drops that are transported in regions of different gas composition. The more volatile species released in the gas phase earlier during the drop lifetime reside in the lower stream while intermediary molar weight species, which egress after the drops are entrained in the mixing layer, reside in the mixing layer and form there a very heterogeneous mixture; the heavier species that evaporate later during the drop lifetime tend to reside in regions of high drop number density. This leads to a segregation of species in the gas phase based on the relative evaporation time from the drops. The ensemble-average drop temperature becomes eventually larger/smaller than the initial drop temperature in MC/SC simulations. Neither this species segregation nor the drop temperature variation with respect to the initial temperature or as a function of the mass loading can be captured by the SC-fuel simulations. (C) 2004 American Institute of Physics.
\end{abstract}

[DOI: $10.1063 / 1.1688327]$

\section{INTRODUCTION}

Most power producing combustion devices employ sprays of commercial petroleum fuels that typically contain hundreds of pure species. Despite the preponderance of multicomponent (MC) fuels, the specific behavior of such sprays in turbulent flows is not well understood when compared to that of single-component (SC) fuel sprays. For example, because of the daunting difficulty of simulating MC mixtures, investigators use a variety of heavy (i.e., large molar weight) SCs or mixtures of two fuels to simulate diesel fuel, ${ }^{1-4}$ al-

a) Telephone: 818-354-6959: fax: 818-393-5011. Electronic mail: josette.bellan@jpl.nasa.gov though there is emerging interest in a complete $\mathrm{MC}$ representation. ${ }^{5}$ Also, because data obtained with SC fuels lead to results that are more easily interpretable, some experiments tend to focus on SC fuels as well, although the ultimate interest is on MC fuels. ${ }^{1}$ The focus of this paper is on $\mathrm{SC}$ versus $\mathrm{MC}$ simulations. The questions we address are as follows: Which of the features of MC two-phase flows with phase change are reproduced by SC flows, and which features, if any, are not? Also, if some features are not reproduced, are they important?

Given the complexities of spatial sprays in combustion chambers, simpler geometric configurations, such as mixing layers, are a reasonable starting point for fundamental stud- 
ies. Moreover, considering the complexities associated with spatial mixing layer boundary conditions, temporal mixing layers are the simplest pertinent configuration. The present study employs direct numerical simulation (DNS) to investigate the behavior of a temporal mixing layer whose lower stream is initially laden with a large number of evaporating MC-fuel drops. DNS studies with solid particles in the absence of phase change were previously performed by Boivin et al. ${ }^{6}$ and by Mashayek and Jaberi ${ }^{7}$ in the context of isotropic turbulence; by Mashayek, ${ }^{8}$ who investigated evaporating drops in isotropic turbulence; by Réveillon and Vervisch, ${ }^{9}$ who studied clusters and randomly distributed evaporating $\mathrm{SC}$-fuel drops in a three-dimensional (3D) freely decaying turbulence; by Mashayek, ${ }^{10}$ who explored evaporating drops in homogeneous shear; and by Miller and Bellan ${ }^{11,12}$ and Okong'o and Bellan, ${ }^{13}$ who studied 3D mixing layers with evaporating SC-fuel drops. The present DNS methodology generally follows that of Refs. 11 and 13 while the drop model is entirely novel in the context of mixing layers. The change in the drop model induces corresponding changes in the mixing layer model. The introduction of the specific MCfuel drop model is motivated by the observation that it may be impractical to model mixtures composed of a large number of species by accounting for each individual constituent (e.g., the single drop, binary-fuel study of Harstad and Bellan ${ }^{14}$ or of Megaridis and Sirignano, ${ }^{15}$ and the MC-fuel drop model of Law and $\mathrm{Law}^{16}$ ). Therefore the adopted strategy is to use the statistical point of view embedded in the continuous thermodynamics (CT) approach. CT is a wellestablished theory with a firm theoretical basis; detailed derivations of a self-consistent theory based on this concept are found in Gal-Or et al., ${ }^{17}$ and in Cotterman et al. ${ }^{18}$ in the context of phase equilibrium calculations. Other derivations and applications are described by Bowman and Edmister, ${ }^{19}$ Edmister and Bowman, ${ }^{20}$ and Briano and Glandt. ${ }^{21}$ The CT theory is based on the appropriate representation of the chemical potential for a mixture containing numerous components and uses molecular thermodynamic methods to represent the Gibbs function in terms of the probability distribution function (PDF) describing the mixture composition. The concepts are fundamental and independent of the physicochemical model chosen to represent the chemical potential. For a specified initial PDF, the evolution of the mixture is governed by thermodynamic relationships and/or conservation equations. Moreover, the $\mathrm{CT}$ approach was validated by many investigators through successful calculations: (i) calculations of vapor-liquid equilibrium (Rätzsch and Kehlen, ${ }^{22}$ Tamim and Hallett ${ }^{23}$ ), (ii) computations of liquidliquid equilibrium (Rätzsch and Kehlen ${ }^{22}$ ), (iii) simulations of polymer solutions (Cotterman et al., ${ }^{18}$ Rätzsch and Kehlen, ${ }^{22}$ Whitson $^{24}$ ), (iv) computations of distillation (Rätzsch and Kehlen ${ }^{22}$ ), (v) flash point calculations (Cotterman et al.,${ }^{18}$ Chou and Prausnitz, ${ }^{25}$ Whitson $^{24}$ ), and (vi) characterization of carbon plus fractions (Whitson ${ }^{24}$ ). Based on the CT approach, Tamim and Hallett ${ }^{23}$ and Hallett ${ }^{26}$ have developed a model for the evaporation of a single, isolated drop of fuel that is a mixture of very many species. Furthermore, the same model has been used by Lippert and Reitz ${ }^{27}$ as a building block in codes devoted to practical applica- tions. The present study adopts the CT approach and utilizes it in a fundamental study of the coupled interaction between a multitude of drops and the flow in a temporal mixing layer at atmospheric pressure.

First, we recall the CT model in the context of a single drop and derive the CT model for a mixing layer. The potential of the CT model is next demonstrated in the context of a single drop by first comparing its predictions with a validated SC-fuel model and then assessing its qualitative behavior for MC fuels. Mixing layer results, focussing on transitional state analysis, are then presented. These encompass global characteristics, detailed visualizations, calculations of probability density functions (PDFs), and first-order statistics. The emerging picture is that of important detailed differences between the predictions of SC- and MC-fuel models. In particular, our results show that the increasing lifetime of the MC drops compared to SC drops results in a more structured flow field, and reveal a molar weight stratification in $\mathrm{MC}$ simulations that inherently cannot be obtained with any SC fuel.

\section{MATHEMATICAL MODEL}

The governing equations are formulated for the temporal mixing layer in an Eulerian-Lagrangian frame for the gas and drops, respectively. This representation is consistent with the volumetrically small loading $\left(\simeq 10^{-3}\right)$, although the mass loading can be substantial due to the very high density ratio between the liquid (subscript $l$ ) and carrier gas, $\rho_{l} / \rho$ $\sim O\left(10^{3}\right)$. Moreover, the drops are treated as point sources of mass, momentum, and energy. This representation is consistent with the drop size being smaller than the Kolmogorov scale (see discussion in Ref. 6). Although the point source representation is no true DNS, this accepted terminology is adopted. Unsteady drag and added mass effects are neglected, as well as Basset history forces, all of which are small for $\rho_{l} / \rho \sim O\left(10^{3}\right)$ (Ref. 6); further neglected in this volumetrically dilute regime are drop collisions and evaporative drop interactions. Moreover, the carrier gas is assumed calorically perfect.

\section{A. Continuous thermodynamics for single MC-fuel drops}

The primary idea of CT modeling is to describe the fuel composition (both liquid and vapor) using a distribution function $f$. Although generally $f$ depends on many parameters representing the characteristics of the fuels, it has been shown ${ }^{18,26}$ that in certain cases it is possible to reduce this dependency to a single parameter: the species molar weight $m$. This simplification is available for mixtures composed of homologous species ${ }^{17,18}$ and includes diesel and gasoline fuels, ${ }^{18,23}$ both of which are of major practical interest. The advantage of such a statistical description is that while a wide range of individual species can be accommodated in the mixture, the number of governing equations is minimally augmented with respect to that necessary for a single species because the composition is represented by a small number of parameters determining $f$. 
In $\mathrm{CT}, f$ is used to define the mole fraction of species $\alpha$, $X_{\alpha}$, whose molar weight lies within the range $m_{\alpha}$ to $m_{\alpha}$ $+\Delta m_{\alpha}$ through

$$
X_{\alpha}=f(m) \Delta m_{\alpha}
$$

with the normalization condition

$$
\int_{0}^{\infty} f(m) d m=1 .
$$

Because mixtures always contain a finite number of individual species, in all CT applications $f$ is non-null only in a finite interval. ${ }^{17}$ Whitson $^{24}$ has shown that gamma distributions may be used to characterize the molar weight of crude oils through

$$
f(m)=\frac{(m-\gamma)^{\zeta-1}}{\beta^{\zeta} \Gamma(\zeta)} \exp \left[-\left(\frac{m-\gamma}{\beta}\right)\right],
$$

where $\Gamma(\zeta)=\int_{0}^{\infty} \xi^{\zeta-1} e^{-\zeta} d \xi$. The origin of $f$ is specified by $\gamma$, and its shape is determined by two parameters, $\zeta$ and $\beta$. These parameters are related to the mean $\theta$, the variance $\sigma^{2}$, and the second moment $\psi$, of $f$ by $\theta=\zeta \beta+\gamma, \sigma^{2}=\zeta \beta^{2}$, and $\psi=\theta^{2}+\sigma^{2}$.

\section{Gas-phase conservation equations}

If the overall vapor mole fraction is $X_{v}$, the carrier gas mole fraction is $X_{g a}=1-X_{v}$, and the vapor phase mole fraction of species $\alpha$ is defined through

$$
X_{\alpha}=X_{v}\left[f_{v}(m)\right] \Delta m_{\alpha},
$$

where $f_{v}$ is the fuel vapor PDF. Multiplying Eq. (1) by $m$ and by $m^{2}$ and integrating it for infinitesimally small $\Delta m_{\alpha}$ yields the mean molar weight of the vapor, $\theta_{v}$ $=\int_{\gamma_{v}}^{\infty} f_{v}(m) m d m$ and the second moment $\psi_{v}$ $=\int_{\gamma_{v}}^{\infty} f_{v}(m) m^{2} d m$, respectively.

In discrete form, the mean molar weight is defined as

$$
m=m_{g a} X_{g a}+\sum_{\alpha=1}^{N} m_{\alpha} X_{\alpha} \quad \alpha \in \text { fuel, }
$$

where $N$ is the total number of fuel species, and the equivalent expression in continuous form is

$$
m=m_{g a}\left(1-X_{v}\right)+\theta_{v} X_{v} .
$$

The gas phase is considered to be a mixture of perfect gases and thus

$$
p=\frac{\rho R_{u} T}{m}=c R_{u} T,
$$

where $\rho=m c$ is the mass density of the gas mixture, $p$ is the thermodynamic pressure, $R_{u}$ is the universal gas constant, $T$ is the temperature, and $c$ is the molar density.

The CT gas-phase conservation equations are derived as in Ref. 23 from the unsteady discrete form of the molar fraction conservation and enthalpy equations,

$\frac{\partial\left(c X_{\alpha}\right)}{\partial t}+\nabla \cdot\left(c X_{\alpha} \mathbf{u}^{*}\right)=\nabla \cdot\left(c D_{\alpha} \nabla X_{\alpha}\right)$,

$$
\begin{aligned}
C_{p v} & \frac{\partial(c T)}{\partial t}+C_{p v} \nabla \cdot\left(c T \mathbf{u}^{*}\right) \\
\quad & \nabla \cdot\left(\lambda_{v} \nabla T\right)-\nabla \cdot\left(\sum_{\alpha=1}^{N} \mathbf{J}_{D \alpha}^{*} h_{v \alpha}\right) .
\end{aligned}
$$

This CT derivation involves (i) substituting $X_{\alpha}$ by $X_{v} f(m)$, integrating Eq. (8) over $d m$ and taking its first two moments, which leads to conservation equations for $c X_{\alpha}, c X_{\alpha} m, c X_{\alpha} m^{2}$, and (ii) integrating Eq. (9) over $d m$ to yield a conservation equation for $c T$. Since a detailed derivation is available, ${ }^{23}$ only a succinct exposition is presented here. In Eqs. (8) and (9), $t$ is the time, $\mathbf{u}^{*}$ is the molar average velocity, $D_{\alpha}$ is the diffusivity of species $\alpha$ in the mixture, $C_{p v}$ is the vapor molar heat capacity at constant pressure, $\lambda$ is the thermal conductivity, $\mathbf{J}_{D \alpha}^{*}=-c D_{\alpha} \nabla X_{\alpha}$ is the diffusional molar flux of species $\alpha$, and $h_{v \alpha}$ is the enthalpy of the $\alpha$ species in the gas phase. Since $\rho_{l} / \rho$ $\sim O\left(10^{3}\right)$, the gas phase is quasisteady with respect to the liquid phase, ${ }^{28}$ and the equations further simplify to yield

$$
\begin{aligned}
\nabla \cdot\left(c X_{v} \mathbf{u}^{*}\right)=\nabla \cdot\left(c \bar{D} \nabla X_{v}\right), \\
\nabla \cdot\left(c X_{v} \theta_{v} \mathbf{u}^{*}\right)=\nabla \cdot\left[c \tilde{D} \nabla\left(X_{v} \theta_{v}\right)\right], \\
\nabla \cdot\left(c X_{v} \psi_{v} \mathbf{u}^{*}\right)=\nabla \cdot\left[c \hat{D} \nabla\left(X_{v} \psi_{v}\right)\right], \\
\bar{C}_{p v} \nabla \cdot\left(c T \mathbf{u}^{*}\right)=\nabla \cdot\left(\lambda_{v} \nabla T\right)+\left[\left(R_{u} A_{c} T-C_{p g a} T\right) c \bar{D}\right. \\
\left.+R_{u} B_{c} c \tilde{D} T \theta_{v}\right] \nabla X_{v},
\end{aligned}
$$

where additional simplifications have been performed by neglecting the difference of terms that are approximately equal. ${ }^{23}$ Several averaging definitions were introduced in Eqs. (10)-(13) using the CT form of the diffusivity, $D(m, T)$, and heat capacity, $C_{p v}(m)$,

$$
\begin{aligned}
& \bar{D}=\int_{\gamma_{v}}^{\infty} D(m, T) f_{v}(m) d m \\
& \widetilde{D} \theta_{v}=\int_{\gamma_{v}}^{\infty} D(m, T) f_{v}(m) m d m \\
& \hat{D} \psi_{v}=\int_{\gamma_{v}}^{\infty} D(m, T) f_{v}(m) m^{2} d m, \\
& \bar{C}_{p v}=X_{v} \int_{\gamma_{v}}^{\infty} C_{p v}(m) f_{v}(m) d m+\left(1-X_{v}\right) C_{p g a},
\end{aligned}
$$

having used, as in Ref. 23, the correlation of Ref. 25,

$$
C_{p v}(m)=R_{u}\left[A_{c}\left(T_{s}\right)+B_{c}\left(T_{s}\right) m\right],
$$

where the subscript $s$ labels the drop surface. Similar to $C_{p v}(m)$, correlations are also available for $D(m, T)=\left(A_{D}\right.$ $\left.+B_{D} m\right) T^{5 / 2} /\left(B_{\Phi}+T\right)$ that can approximate the diffusional behavior of the $\alpha$ species in the mixture; constants $A_{D}, B_{D}$, and $B_{\Phi}$ are listed in Ref. 23.

\section{Liquid-phase conservation equations}

The conservation equations for the liquid phase are obtained under the assumption of a well-mixed liquid, meaning 
that internal circulation is very effective and renders the properties of the drop uniform in a time much shorter than the drop lifetime. This assumption is consistent with the slow evaporation limit (see Makino and $\mathrm{Law}^{29}$ ) of the present mixing layer simulations. Departures from this well-mixed state are expected to become increasingly important with wider separation of the saturation vapor-pressure curves for different species, however, for a continuous mixture these departures may be considerably reduced. Following the CT derivation and approximations of Ref. 23, including the assumption of constant $\rho_{l}$, the conservation equations for a spherically symmetric drop are

$$
\begin{aligned}
& J_{r s}^{*}\left(1-X_{v s}\right)=-c \bar{D}\left(\nabla X_{v}\right)_{s}, \\
& \frac{d \theta_{l}}{d t}=\frac{6}{c_{l} d}\left[J_{r}^{*}\left(\theta_{l}-\theta_{v} X_{v}\right)+c \widetilde{D} \nabla\left(X_{v} \theta_{v}\right)\right]_{s}, \\
& \frac{d \psi_{l}}{d t}=\frac{6}{c_{l} d}\left[J_{r}^{*}\left(\psi_{l}-\psi_{v} X_{v}\right)+c \hat{D} \nabla\left(X_{v} \psi_{v}\right)\right]_{s}, \\
& \frac{d T_{d}}{d t}=\frac{6}{C_{p l} c_{l} d}\left[q_{s}-J_{r s}^{*} L_{v}\right],
\end{aligned}
$$

where $J_{r s}^{*}=\left(d \mathcal{N}_{d} / d t\right) / A$ is the drop-surface radial molar flux (subscript $d$ refers to the drop), $\mathcal{N}_{d}=M_{d} / \theta_{l}$ is the number of moles in the drop, $M_{d}$ is the drop mass flux, $A=\pi d^{2}$ is the drop area, $d$ is the drop diameter, $c_{l}$ is the liquid molar density $\left(c_{l}=\rho_{l} / \theta_{l}\right), C_{p l}$ is the liquid heat capacity at constant pressure, $q_{s}$ is the drop surface heat flux, and $L_{v}$ is the liquid latent heat. The rapid mixing assumption implies that $T_{d}$ $=T_{s}$.

\section{Boundary conditions}

The boundary conditions are applied both in the drop far field (subscript $e$ ) and at the drop surface, with consistent phase coupling. The far-field values of the dependent variables are specified through the given gas composition and temperature.

To ensure consistency in the definition of gas and liquid enthalpies $h_{v}$ and $h_{l}$, it is recalled that for calorically perfect species

$$
h_{l}=\int_{0}^{T} C_{p l} d T^{\prime}, \quad h_{v}=\int_{0}^{T} C_{p v} d T^{\prime}+h_{v}^{0},
$$

where the molar enthalpy of the mixture in continuous form is

$$
h=\left(1-X_{v}\right) C_{p g a} T+X_{v}\left(C_{p v} T+h_{v}^{0}\right) .
$$

The reference enthalpy for the liquid and the carrier gas are taken to be null at $T=0$, and a non-null reference value of the enthalpy of the vapor mixture, $h_{v}^{0}$, is required for the vapor. To find $h_{v}^{0}$, its relation to $L_{v}$ is derived, having defined

$$
\mathbf{J}^{*} L_{v}=\sum_{\alpha=1}^{N} \mathbf{J}_{\alpha}^{*} L_{v \alpha}
$$

where $\mathbf{J}^{*}$ is the total molar flux and $\mathbf{J}_{\alpha}^{*}=\mathbf{J}^{*} X_{\alpha}+\mathbf{J}_{D \alpha}^{*}$. For each component

$$
L_{v \alpha}=h_{v \alpha}\left(T_{s}\right)-h_{l \alpha}\left(T_{s}\right),
$$

where $\quad h_{v \alpha}\left(T_{s}\right)=h_{v \alpha}^{0}+\int_{0}^{T_{s}} C_{p v \alpha} d T^{\prime} \quad$ and $\quad h_{l \alpha}\left(T_{s}\right)$ $=\int_{0}^{T_{s}} C_{p l \alpha} d T^{\prime}$. Substituting Eq. (18) and those in Ref. 23 for $C_{p l}\left(\theta_{l}\right)$ and $L_{v}\left(T_{s}, \theta_{v s}\right)$ in Eq. (25) and integrating the righthand side over all species yields an equation with a single unknown $h_{v}^{0}$, for which the equation is solved. Thus, following Ref. 23, in discrete form, $L_{v}(m)=\left(A_{h}+B_{h} m\right) \phi_{h}$ with $\phi_{h}=\left[\left(T_{c r}-T_{s}\right) /\left(T_{c r}-T_{b}\right)\right]^{0.38}$, and the equivalent CT form is obtained by integrating over $m$ as

$$
\begin{aligned}
& C_{p l}=\theta_{l}\left[A_{l}+B_{l} T_{d}+C_{l} T_{d}^{2}\right], \\
& L_{v}\left(T_{s}\right)=\phi_{h} \times\left[A_{h}+B_{h} X_{v s} \theta_{v s}-\frac{B_{h} c \bar{D}}{J_{r s}}\left[\nabla\left(X_{v} \theta_{v}\right)\right]_{r, s}\right],
\end{aligned}
$$

where $A_{l}, B_{l}, C_{l}, A_{h}$, and $B_{h}$ are constants listed in Ref. 23 and the subscripts $b$ and $c r$ refer to the boiling point and to the critical point. Also, in discrete form, $T_{c r}(m)=A_{c r}$ $+B_{c r} m$ and the equivalent $\mathrm{CT}$ form is obtained by integrating over $m$. For consistency with other correlations, it can be shown that a linear approximation of $h_{v}^{0}(m)=K m+K^{\prime}$ can be made, and an equivalent CT expression is used in the calculation (see Sec. IV A). Since for a specified fuel $h_{v}^{0}$ is constant, it is evaluated at the reference temperature $T_{w b}$. To compute $T_{w b}$, an empirical correlation of experimental results is employed, ${ }^{30}$

$$
T_{w b}=137\left(\frac{T_{b}}{373.15}\right)^{0.68} \log _{10}(T)-45,
$$

where in discrete form $T_{b}(m)=A_{b}+B_{b} m$ and the equivalent CT expression is used in Eq. (29); $A_{b}$ and $B_{b}$ are constants whose values are listed in Ref. 23.

The boundary conditions at the drop surface express the conservation of total (i.e., diffusive plus convective) molar mass, molar species, and heat fluxes. Raoult's law relates the fugacities across the drop surface, leading for a mixture of discrete components to

$$
X_{v \alpha}=X_{l \alpha}\left(p_{v \alpha} / p\right),
$$

where $p_{v \alpha}$ is the $\alpha$-species vapor pressure. If $f_{l}$ is the liquid composition PDF, in CT form, Raoult's law becomes

$$
X_{v}=\int_{\gamma_{l}}^{\infty} f_{l}(m) \frac{p_{v}(m)}{p} d m,
$$

which weighted by $m$ and $\left(m-\theta_{v}\right)^{2}$ yields the vapor mean molar weight and the vapor variance in molecular weight at drop surface,

$$
\begin{aligned}
& \left(X_{v} \theta_{v}\right)_{s}=\int_{\gamma_{l}}^{\infty} f_{l}(m) \frac{p_{v}(m)}{p} m d m, \\
& \left(X_{v} \sigma_{v}^{2}\right)_{s}=\int_{\gamma_{l}}^{\infty} f_{l}(m) \frac{p_{v}(m)}{p}\left(m-\theta_{v}\right)^{2} d m .
\end{aligned}
$$

The vapor pressure is given by the Clausius-Clapeyron equation in CT form, 


$$
p_{v}(m)=p_{a t m} \exp \left[\left(\frac{\Delta s_{f g}(m)}{R_{u}}\right)\left(1-\frac{T_{b}(m)}{T_{s}}\right)\right],
$$

where $p_{\text {atm }}=1 \mathrm{~atm}$ and the entropy of vaporization $\Delta s_{f g}(m)$ can be expressed using Trouton's empirical law $\Delta s_{f g}(m)$ $=L_{v}(m) / T_{b}(m) \simeq 87.9 \mathrm{~J} \mathrm{~K}^{-1} \mathrm{~mol}^{-1}$. Thus Eqs. (31)-(34) determine the drop surface conditions in the vapor phase, which drive evaporation. Using the relationships between $\theta$, $\zeta, \beta$, and $\gamma$, assuming $\gamma_{l}=\gamma_{v s}=\gamma$ and integrating Eqs. (31)(34) over $m$ to obtain the CT form of the boundary conditions yields relationships between $X_{v s}$ and the distribution parameters in the liquid, and between the distribution parameters in the liquid and vapor,

$$
\begin{aligned}
& X_{v s}=\frac{p_{a t m}}{p_{e}} \frac{\exp \left[\Delta s_{f g} /\left(R_{u} T_{s}\right)\left(T_{s}-A_{b}-\gamma B_{b}\right)\right]}{\left[1+\Delta s_{f g} /\left(R_{u} T_{s}\right) B_{b} \beta_{l}\right]^{\zeta_{l}}}, \\
& \theta_{v s}-\gamma=\frac{\theta_{l}-\gamma}{1+\frac{\left(\Delta s_{f g} / R_{u}\right)\left(1-A_{b} / T_{s}\right)\left[B_{b} /\left(T_{s}-A_{b}\right)\right] \sigma_{l}^{2}}{\theta_{l}-\gamma}} \\
& \sigma_{v s}^{2}=\sigma_{l}^{2}\left[\frac{\theta_{s v}-\gamma}{\theta_{l}-\gamma}\right]^{2} .
\end{aligned}
$$

\section{B. Mixing layer conservation equations}

\section{Gas-phase conservation equations}

The gas-phase formulation of Ref. 11 is here modified in three ways. First, two transport equations, for $\theta_{v}$ and $\psi_{v}$, are added to represent the entire molar weight range of evaporated fuel species; $\psi_{v}$ is used as a dependent variable instead of $\sigma_{v}^{2}$ because the resulting equation is simpler. Second, as in Ref. 13, the influence of the species-mass diffusion velocities is included in the heat flux vector because they were shown in Ref. 13 to be the dominant contribution, as conductive effects were relatively small in these simulations. Finally, since all available thermophysical property correlations ${ }^{25,23}$ utilize $m_{\alpha}$, for consistency, two of the primitive variables are now $c$ and $X_{\alpha}$ instead of $\rho$ and the mass fractions, $Y_{\alpha}$ $=X_{\alpha} m_{\alpha} / m$ :

Continuity.

$$
\frac{\partial \rho}{\partial t}+\frac{\partial}{\partial x_{j}}\left(\rho u_{j}\right)=S_{I \text {-mass }},
$$

where $j$ denotes the Cartesian coordinate, $\mathbf{u}$ is the velocity of the mean mass, and $S_{I \text {-mass }}$ is the mass source due to evaporation. This leads to an equation for $c$,

$$
\frac{\partial c}{\partial t}+\frac{\partial}{\partial x_{j}}\left(c u_{j}\right)=-\frac{c}{m} \frac{D m}{D t}+\frac{S_{I-\text { mass }}}{m} .
$$

Further manipulation of Eq. (39) with the $\theta_{v}$ equation (developed below) yields

$$
\begin{aligned}
\frac{\partial c}{\partial t}+ & \frac{\partial}{\partial x_{j}}\left(c u_{j}\right) \\
= & \frac{\partial}{\partial x_{j}}\left(c \bar{D} \frac{\partial}{\partial x_{j}}\left(X_{v}\right)\right)-\frac{1}{m_{g a}} \frac{\partial}{\partial x_{j}}\left(c \widetilde{D} \frac{\partial}{\partial x_{j}}\left(X_{v} \theta_{v}\right)\right) \\
& +S_{I \text {-mole }}
\end{aligned}
$$

where $S_{I \text {-mole }}$ is the molar source due to evaporation.

Momentum conservation.

$$
\frac{\partial\left(\rho u_{i}\right)}{\partial t}+\frac{\partial}{\partial x_{j}}\left(\rho u_{i} u_{j}+p \delta_{i j}-\tau_{i j}\right)=S_{I I, j},
$$

where $\tau_{i j}$ is the stress tensor,

$$
\begin{aligned}
\tau_{i j} & =\mu\left[2 S_{i j}-(2 / 3) S_{k k} \delta_{i j}\right], \\
S_{i j} & =(1 / 2)\left(\partial u_{i} / \partial x_{j}+\partial u_{j} / \partial x_{i}\right),
\end{aligned}
$$

with $\delta_{i j}$ being the Kronecker symbol, and $\mathbf{S}_{I I}$ is the drop-gasinteraction momentum source.

Energy conservation.

$$
\begin{gathered}
\frac{\partial\left(c e_{t}\right)}{\partial t}+\frac{\partial}{\partial x_{j}}\left[\left(c e_{t}+p\right) u_{j}-\lambda \frac{\partial T}{\partial x_{j}}-u_{i} \tau_{i j}\right] \\
+\frac{\partial}{\partial x_{j}}\left(\sum_{\beta=[g a,[1, N]]} J_{D j \beta} h_{\beta}\right)=S_{I I I},
\end{gathered}
$$

where $S_{I I I}$ is the drop-gas-interaction source term and

$$
\begin{aligned}
& \sum_{\beta=[g a,[1, N]]} J_{D j \beta} h_{\beta} \\
& =-\left(\int_{\gamma_{v}}^{\infty} c D(m) \frac{\partial\left[X_{v} f_{v}(m)\right]}{\partial x_{j}}\left[h_{v}^{0}(m)+C_{p v}(m) T\right] d m\right) \\
& \quad+J_{D j g a} C_{p g a} T
\end{aligned}
$$

where $\mathbf{J}_{D}$ is the mass diffusion flux. Since $\Sigma_{\beta=[g a,[1, N]]^{m}} \mathbf{J}_{D \beta}=0$, it follows that

$$
J_{D j g a}=\frac{1}{m_{g a}} \int_{\gamma_{v}}^{\infty} c m D(m) \frac{\partial\left[X_{v} f_{v}(m)\right]}{\partial x_{j}} d m,
$$

leading to

$$
\begin{aligned}
\sum_{\beta=[g a,[1, N]]} J_{D j \beta} h_{\beta} & \\
= & -\left(K^{\prime}+R_{u} A_{c} T\right) c \bar{D} \frac{\partial X_{v}}{\partial x_{j}} \\
& -\left(K+R_{u} B_{c} T-\frac{C_{p g a} T}{m_{g a}}\right) c \widetilde{D} \frac{\partial\left(X_{v} \theta_{v}\right)}{\partial x_{j}} .
\end{aligned}
$$

Species conservation. The discrete form of the conservation equation for the partial species density is

$$
\frac{\partial\left(\rho Y_{\alpha}\right)}{\partial t}+\frac{\partial\left(\rho Y_{\alpha} u_{j}\right)}{\partial x_{j}}=\frac{\partial}{\partial x_{j}}\left(\rho D_{\alpha} \frac{\partial Y_{\alpha}}{\partial x_{j}}\right)+S_{I \alpha \text {-mass }},
$$

where $S_{I \alpha \text {-mass }}$ is the evaporated mass of $\alpha$ species from the drop; $S_{I \text {-mass }}=\sum_{\alpha=1}^{N} S_{I \alpha \text {-mass }}$. Following the single-drop protocol and assumptions, in CT form this equation becomes

$$
\frac{\partial\left(c X_{v}\right)}{\partial t}+\frac{\partial}{\partial x_{j}}\left(c X_{v} u_{j}-c \bar{D} \frac{\partial X_{v}}{\partial x_{j}}\right)=S_{I \text {-mole }} .
$$

Vapor mean-molar-weight transport equation. Integrating Eq. (47) over $d m$ and using the assumptions of Ref. 23 yields 


$$
\frac{\partial\left(c X_{v} \theta_{v}\right)}{\partial t}+\frac{\partial}{\partial x_{j}}\left[c X_{v} \theta_{v} u_{j}-c \widetilde{D} \frac{\partial\left(X_{v} \theta_{v}\right)}{\partial x_{j}}\right]=S_{I \text {-mass }} .
$$

Second moment transport equation. Multiplying Eq. (47) by $m$ and integrating it over $d m$ leads to

$$
\frac{\partial\left(c X_{v} \psi_{v}\right)}{\partial t}+\frac{\partial}{\partial x_{j}}\left[c X_{v} \psi_{v} u_{j}-c \hat{D} \frac{\partial\left(X_{v} \psi_{v}\right)}{\partial x_{j}}\right]=S_{\psi},
$$

where $S_{\psi}$ is the source of $c X_{v} \psi_{v}$ in the gas phase due to drop evaporation.

Equation of state. The perfect gas equation of state, $p$ $=c R_{u} T$, closes the system of gas-phase equations.

\section{Individual drop governing equations}

Coupled to the gas-phase conservation equations, the drop equations for the position, $\chi$, the velocity, $\mathbf{v}, T_{d}, \theta_{l}$, and $\psi_{l}$ are

$$
\begin{aligned}
& \frac{d \chi_{i}}{d t}=v_{i}, \\
& \frac{d v_{i}}{d t}=\frac{F_{i}}{M_{d}}, \\
& \frac{d T_{d}}{d t}=\frac{q_{s} A+(d \mathcal{N} / d t) L_{v}}{\mathcal{N} C_{p l}}, \\
& \frac{d \theta_{l}}{d t}=\frac{6 J_{r s}}{c_{l} d}\left[\theta_{l}+\frac{\theta_{v} X_{v}-\theta_{v s} X_{v s}(1+B)}{B}\right], \\
& \frac{d \psi_{l}}{d t}=\frac{6 J_{r s}}{c_{l} d}\left[\psi_{l}+\frac{\psi_{v} X_{v}-\psi_{v s} X_{v s}(1+B)}{B}\right],
\end{aligned}
$$

where, according to Ref. 26,

$$
J_{r s}=\frac{c \bar{D} \mathrm{Sh}}{d} \ln (1+B) \quad \text { with } B=\frac{X_{v s}-X_{v}}{1-X_{v s}},
$$

where $B$ is the CT equivalent of the Spalding transfer number. ${ }^{28}$ The force term $F_{i}$, the heat transfer term $q_{s} A$, and the enthalpy associated with evaporation, $L_{v}(d \mathcal{N} / d t)$, account for the coupling between gas and drops. The values of the gas-phase variables $\left(u_{i}, T, X_{v}, \theta_{v}, \psi_{v}\right)$ at each drop location serve now as the far-field boundary conditions for the single-drop equations presented above. Using the validated models for $F_{i}, q_{s} A$, and $d \mathcal{N} / d t$ described in Refs. 30 and 26 , one obtains

$$
\begin{aligned}
& F_{j}=\left(\frac{M_{d}}{\tau_{d}}\right) f_{1}\left(u_{j}-v_{j}\right), \\
& q_{s} A=\left(\frac{M_{d}}{\tau_{d}}\right) \frac{\mathrm{Nu} C_{p g}}{3 \operatorname{Pr} m} f_{2}\left(T-T_{d}\right), \\
& \frac{d \mathcal{N}}{d t}=J_{r s} \pi d^{2}=-\left(\frac{M_{d}}{\tau_{d}}\right) \frac{\mathrm{Sh}}{3 \operatorname{Sc} m} \ln (1+B),
\end{aligned}
$$

where $\tau_{d}=\rho_{l} d^{2} /(18 \mu)$ is the particle time constant for Stokes flow, and $\mu$ is the viscosity of the carrier gas; $\operatorname{Pr}$ $=\mu C_{p g} /(\lambda m)$ and $\mathrm{Sc}=\mu /(\rho \bar{D})$ are the Prandtl and the Schmidt numbers, respectively. The Nusselt $\mathrm{Nu}$ and the
Sherwood Sh numbers are semiempirically modified using the Ranz-Marshall correlations to account for convective effects in the heat and the mass transfer ${ }^{11}$ and the similarity assumption applies,

$$
\begin{aligned}
& \mathrm{Nu}=2+0.552 \operatorname{Re}_{s l}^{1 / 2}(\operatorname{Pr})^{1 / 3}, \\
& \mathrm{Sh}=2+0.552 \operatorname{Re}_{s l}^{1 / 2}(\mathrm{Sc})^{1 / 3} .
\end{aligned}
$$

$f_{1}$ is an empirical correction to Stokes drag accounting for both finite droplet Reynolds numbers [slip Reynolds number $\operatorname{Re}_{s l}=\|\mathbf{u}-\mathbf{v}\| \rho d / \mu$ where $(\mathbf{u}-\mathbf{v})$ is the slip velocity] and a Reynolds number based on the blowing velocity $\left(\operatorname{Re}_{b}\right.$ $=\rho U_{b} d / \mu$, with $\left.U_{b}=J_{r s} / c\right)$ due to evaporation,

$$
\begin{aligned}
& f_{1}=\frac{1+0.0545 \mathrm{Re}_{s l}+0.1 \mathrm{Re}_{s l}^{1 / 2}\left(1-0.03 \mathrm{Re}_{s l}\right)}{1+a\left|\mathrm{Re}_{b}\right|^{b}}, \\
& a=0.09+0.077 \exp \left(-0.4 \mathrm{Re}_{s l}\right), \\
& b=0.4+0.77 \exp \left(-0.04 \mathrm{Re}_{s l}\right) .
\end{aligned}
$$

The correlation of Eq. (55) is valid for the ranges $0 \leqslant \mathrm{Re}_{s l}$ $\leqslant 100$ and $0 \leqslant \operatorname{Re}_{b} \leqslant 10 . f_{2}$ is an analytical correction to heat transfer due to evaporation,

$$
f_{2}=\frac{\kappa}{\exp (\kappa)-1}, \quad \kappa=-1.5 \operatorname{Pr} \tau_{d} \frac{1}{\mathcal{N}} \frac{d \mathcal{N}}{d t} .
$$

Finally, $\mu$ is computed from the specified initial (subscript 0 ) Reynolds number $\operatorname{Re}_{0}$,

$$
\mu=\rho \Delta U_{0} \delta_{\omega, 0} / \operatorname{Re}_{0},
$$

where $\Delta U_{0}=2 U_{0}$ is the initial difference in the free-stream velocities calculated from the specified initial Mach number $M_{c, 0}$ (details in Ref. 11) and $\delta_{\omega, 0}$ is the initial vorticity thickness. The specification of Pr, Sc, and $\operatorname{Re}_{0}$ leads to a family of gas-phase solutions that is independent of the actual values of $\mu, \lambda$, and $\bar{D}$; this is the principle of flow similarity lucidly stated by Batchelor. ${ }^{31}$ However, the drop characteristic time $\tau_{d}$ depends explicitly on $\mu$, meaning that the $\mu$ magnitude will influence the drop interaction with the flow. The choice of the $\tau_{d}(\mu)$ value is intended to render the drop and flow characteristic times of same order of magnitude so as to enable the investigation of their interaction.

\section{Source terms}

The source terms in Eqs. (40), (41), (43), and (48)-(50) express the phase coupling of molar mass, momentum, energy, mean molar weight, and second moment of the distribution function. These source terms originate at the Lagrangian drop locations, but they are needed at the Eulerian grid nodes to express the coupling between phases. Although in reality there is a lag between the time at which the source terms are created at the drop locations and that at which they arrive at the grid nodes, in the point-source DNS concept, this time lag cannot be taken into account. This is because modeling these time-lag subgrid processes would be tantamount to adding a subgrid scale model, and thus this computation could no longer qualify as DNS by the meaning of Boivin et ll $^{6}$ since modeling would then be done in the gas phase away from the vicinity of the drops and the arguments 
of the point-source model where only the immediate vicinity (a sphere of influence around the drop) is modeled and all other scales of the flow are resolved, would no longer hold. Thus the state-of-the-art in SC-fuel DNS of two-phase flows is to add these source terms to the grid nodes proportionally to the drop distance from the eight adjacent grid nodes (e.g., Ref. 13). For MC-fuel point-source DNS, the question arises as to the additional approximations that could be necessary to project the source terms from the drop locations to the grid nodes. Succinctly, this question can be addressed by examining the characteristic time of source arrival at the nodes. Similar to SC-fuel simulations, in MC-fuel computations, the fluxes of mass, momentum, and energy transport sources from the drop locations to the grid nodes, and each flux is composed of a convective term and a diffusive term. Irrespective of the specific flux, convective contributions have a much faster time scale than the diffusive contributions, and thus the source terms will arrive from the drop locations to the nodes at the convective flux characteristic time. Concerning the approximations made regarding the diffusive fluxes, comparisons between the SC and MC situation reveal that in the former case, the $3 \times 3$ diffusion matrix for the three species system (fuel, oxygen, and nitrogen) is approximated by a single, effective diffusion coefficient, and equivalently, for the MC case, the much larger diffusion matrix is also approximated by a single diffusion coefficient. In both situations, this diffusion matrix approximation has only minor impact on the arrival time of the drop source terms at the grid nodes, since as already stated, the convective fluxes have the fastest characteristic time. Therefore the same strategy as in SC simulations ${ }^{11}$ is here employed to calculate the source terms at the grid nodes: a geometric weighting factor $w_{q}$ is used to distribute the individual drop contributions to the nearest eight grid points in proportion to their distance from the drop location. Using conservation principles, one obtains

$$
\begin{aligned}
& S_{I-\text { mass }}=-\sum_{q=1}^{N_{d}} \frac{w_{q}}{\Delta x^{3}}\left[\frac{d\left(\mathcal{N} \theta_{l}\right)}{d t}\right]_{q}, \\
& S_{I-\text { mole }}=-\sum_{q=1}^{N_{d}} \frac{w_{q}}{\Delta x^{3}}\left[\frac{d \mathcal{N}}{d t}\right]_{q}, \\
& S_{I I, j}=-\sum_{q=1}^{N_{d}} \frac{w_{q}}{\Delta x^{3}}\left[F_{j}+\frac{d\left(\mathcal{N} \theta_{l}\right)}{d t} v_{j}\right]_{q}, \\
& S_{I I I}=-\sum_{q=1}^{N_{d}} \frac{w_{q}}{\Delta x^{3}}\left[v_{j} F_{j}+q_{s} A+\frac{d \mathcal{N}}{d t}\left(\frac{\theta_{l} v_{j} v_{j}}{2}+h_{v, s}\right)\right]_{q}, \\
& S_{\psi}=-\sum_{q=1}^{N_{d}} \frac{w_{q}}{\Delta x^{3}}\left[\frac{d\left(\mathcal{N} \psi_{l}\right)}{d t}\right]_{q}
\end{aligned}
$$

where the summations are over all drops residing within a local numerical discretization volume $\Delta x^{3} \cdot h_{v, s}=C_{l} T_{s}+L_{v}$ is the enthalpy of the evaporated species.

\section{SINGLE-DROP RESULTS}

Before undertaking DNS of the mixing layer with MCfuel drops it is important to assess the ability of the CT method to portray a variety of fuel mixtures. In this spirit, since validated single-SC-fuel drop models do exist, ${ }^{30}$ those results are compared with results from simulations using a sharply peaked distribution $f$. Further, MC-fuel single-drop simulations are conducted to explore the novel potential of the CT formulation.

All single, isolated drop results were calculated by solving Eqs. (20)-(22) and (52) in which $X_{v}$ is replaced by $X_{v e}$ to account for the specified far-field conditions. These equations were solved in conjunction with the boundary conditions of Eqs. (35)-(37) using a finite difference time discretization with a time step of $10^{-6} \mathrm{~s}$. In the MC simulations, all transport properties (including $\lambda$ whose dependency on these variables is listed in Tamim and Hallett ${ }^{23}$ ) were functions of $\theta$ and $T$ as stated above. The values of $\zeta$ and $\beta$ are those of Hallett. $^{26}$

\section{A. Sharply peaked distribution versus SC-fuel representation}

Displayed in Fig. 1 are results obtained from several drop models exercised for the same initial conditions: $T_{e}$ $=1000 \mathrm{~K}, T_{d, 0}=300 \mathrm{~K}, d_{0}=2 \times 10^{-3} \mathrm{~m}, \operatorname{Re}_{d, 0}=17$. The specific $T_{e}$ is chosen because it was shown in Miller et al. ${ }^{30}$ that variations among several drop model predictions emerge only when the evaporation rate is increased. The model which forms the base of the present drop formulation distinguished itself from other models discussed in detail in Ref. 30 by agreeing with the data of Wong and $\operatorname{Lin}^{32}$ (both drop size and temperature evolution) which was for $d_{0}=2$ $\times 10^{-3} \mathrm{~m}$ and $n$-decane at $T_{e}=1000 \mathrm{~K}$ (Fig. 4 in Ref. 30). Here, Model 1 is the rapid mixing model (i.e., the infinite $\lambda_{l}$ limit) without evaporative correction to heat transfer as in Chen and Pereira ${ }^{33}$ (i.e., $f_{2}=1$ ); Model 2 is the rapid mixing model with an evaporative correction to heat and mass transfer as in Abramzon and Sirignano; ${ }^{34}$ Model 3 is based on the heat-mass analogy and like Model 2 takes into account the heating period of the droplet; and Model 4, whose results were found closest to experimental observations in Ref. 30, additionally incorporates the nonequilibrium evaporation law based on the Langmuir-Knudsen (LK) law. Although the present drop model is strictly valid only for slow evaporation, the agreement between simulations using this drop model with experimental data at $T_{e}=1000 \mathrm{~K}$, where evaporation is considerably faster, ${ }^{30}$ indicated that the uniformity assumption for the variables inside the drop may be valid beyond the original regime in which the model was derived, and justified the present comparisons. The MC model is implemented with $\theta_{l, 0}=142 \mathrm{~kg} / \mathrm{kmole}$ to duplicate the molar weight of $n$-decane, $\sigma_{l, 0}^{2}=2(\mathrm{~kg} / \mathrm{kmole})^{2}$ to have a very sharply peaked distribution, and $\gamma_{l, 0}=138 \mathrm{~kg} / \mathrm{kmole}$ to restrict the molar weight of components so as to be close to $n$-decane. The thermophysical properties used in the calculations for $n$-decane are those listed in Ref. 30.

Except for the heating period, the evaporation model using CT is closest to Model 4 (both $d$ and $T_{d}$ evolution) and is 
(a)

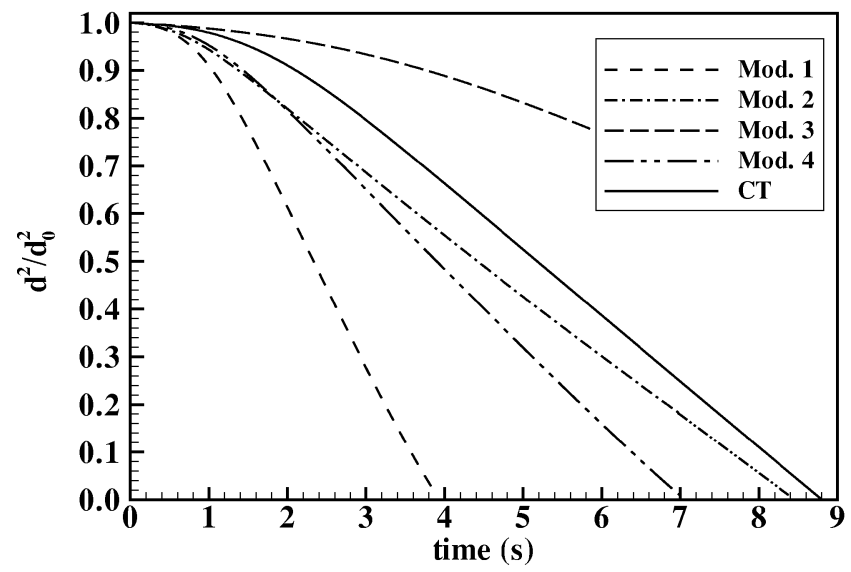

(b)

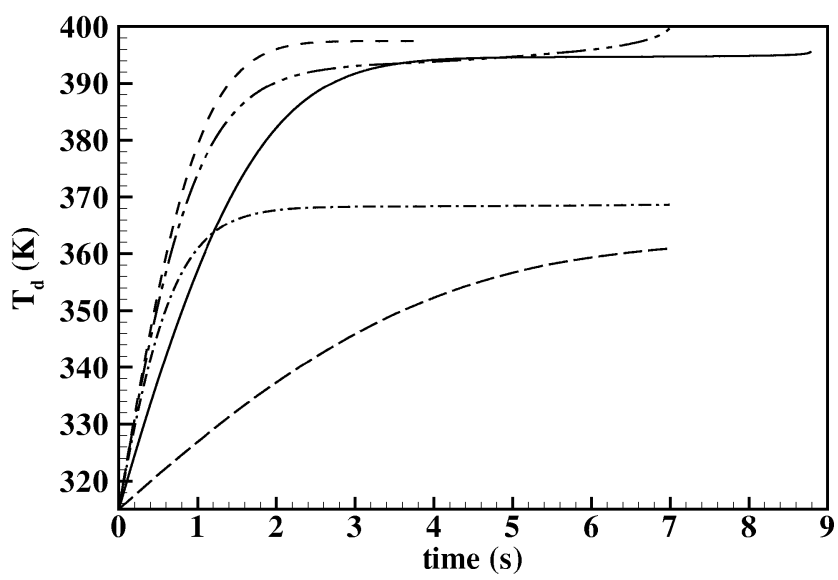

FIG. 1. Drop evaporation-model comparison. Initial conditions are $T_{e}$ $=1000 \mathrm{~K}, T_{d, 0}=300 \mathrm{~K}, d_{0}=2 \times 10^{-3} \mathrm{~m}$, and $\mathrm{Re}_{d, 0}=17$, (a) normalized residual drop area, (b) drop temperature.

in better agreement with it than most of the other models, which are typically employed in two-phase flow simulations with evaporating drops. This agreement with a validated model is significant. The slight difference in the heating period between Model 4 and the CT model is attributed to the corresponding difference in fuel composition between the MC fuel and $n$-decane, which is reflected in the thermophysical properties. We also note that LK effects are insignificant for $d_{0}=2 \times 10^{-3} \mathrm{~m}$, and thus are not expected to influence the results (Model 4 includes LK effects, whereas the CT-based model does not). Based on the comparison presented in Fig. 1, the CT model seems to combine quantitative accuracy (given the difference in composition between the constant SC molar weight and the sharply peaked- $f$ MC equivalent) with a more realistic description of the drop physics.

\section{B. Single-MC-fuel drop results}

To establish the difference in baseline behavior between different MC fuels, several MC-fuel drop calculations are conducted. The parameters characterizing $f$ for all the fuels used in the computations are listed in Table I. The values of $\theta_{l, 0}$ and $\sigma_{l, 0}$ are prescribed, whereas $\gamma_{l, 0}$ is calculated from
TABLE I. Parameters characterizing the density and distribution function for different fuels. The density is in $\mathrm{kg} / \mathrm{m}^{3}$ and all parameters related to the distribution function are in $\mathrm{kg} / \mathrm{kmole}$.

\begin{tabular}{lcccc}
\hline \hline Fuel & $\rho_{l}$ & $\theta_{l, 0}$ & $\sigma_{l, 0}$ & $\gamma_{l, 0}$ \\
\hline$n$-decane & 642 & 142 & & \\
Gasoline & 742 & 101 & 31.5 & 60.5 \\
Diesel & 828 & 185 & 43.0 & 60.5 \\
\hline \hline
\end{tabular}

the condition that $T_{d, 0}<T_{b, 0}$ and therefore $\gamma_{l, 0}=\left(T_{d, 0}\right.$ $\left.-A_{b}\right) / B_{b}$, meaning that it corresponds to the species having the lowest boiling point.

The results plotted in Fig. 2 are all for $T_{e}=1000 \mathrm{~K}$, $T_{d, 0}=300 \mathrm{~K}, \operatorname{Re}_{d, 0}=0$, and $d_{0}=10^{-4} \mathrm{~m}$. For diesel, which is the least volatile fuel, an enlarged heating period is necessary before initiating vaporization, and thus the drop lifetime [Fig. 2(a)] is largest. The heating period of gasoline is slightly shorter than that of $n$-decane because the lighter species in gasoline are more volatile, however, as the heavier species begin evaporating, the drop evaporation rate decreases with respect to $n$-decane. Illustrated in Fig. 2(b) is the time evolution of $T_{d}$ and $T_{b}$ for the $n$-decane and dieselfuel drops. In both situations, $T_{d}$ is bounded by $T_{b}$; for $n$-decane, an asymptotic $T_{d}$ behavior is reached, however, no such situation occurs for the diesel drop whose $T_{b}$ evolves with the composition. Comparing the initial diesel-fuel drop composition with that at half through the drop lifetime [Fig. 2(c)], one discerns the disappearance of the lighter molar weight species, which are well known to be more volatile, and the concomitant larger peak at a larger molar weight. To further quantify the diesel-fuel drop evolution, the surface vapor mole fraction is displayed in Fig. 2(d) as a function of $t$. Following the initial transient during which $X_{v s}$ continuously increases, a stationary state is reached corresponding to a quasisteady evaporation.

\section{MIXING LAYER RESULTS}

To explore the approximations introduced by the $\mathrm{SC}$ assumption, DNS of SC- and MC-fuel drop-laden mixing layers are compared for the same conditions except for the identity of the fuel in the drops. The present MC simulations using diesel fuel are compared with those of Okong'o and Bellan ${ }^{13}$ using $n$-decane (a SC simulant of diesel fuel). The free-stream carrier gas temperature $T_{0}$ is relatively low to ensure that the characteristic times of the drops and flow are of the same order of magnitude at the present Reynolds number values which are smaller than in fully turbulent flows.

\section{A. Numerical procedure, initial and boundary conditions}

Figure 3 shows the computational domain configuration and the definition of the streamwise, $x_{1}$, cross-stream, $x_{2}$, and spanwise, $x_{3}$, coordinates with lengths $L_{1}=4 \lambda_{1}$ $=29.16 \delta_{\omega, 0}, L_{2}=1.1 L_{1}$, and $L_{3}=4 \lambda_{3}=0.6 L_{1}$, with $L_{1}$ $=0.2 \mathrm{~m}$. The parameters $\lambda_{1}$ and $\lambda_{3}$ are forcing wavelengths in the $x_{1}$ and $x_{3}$ directions, and were used to excite the layer in order to induce rollup and pairing as in Moser and 
(a)

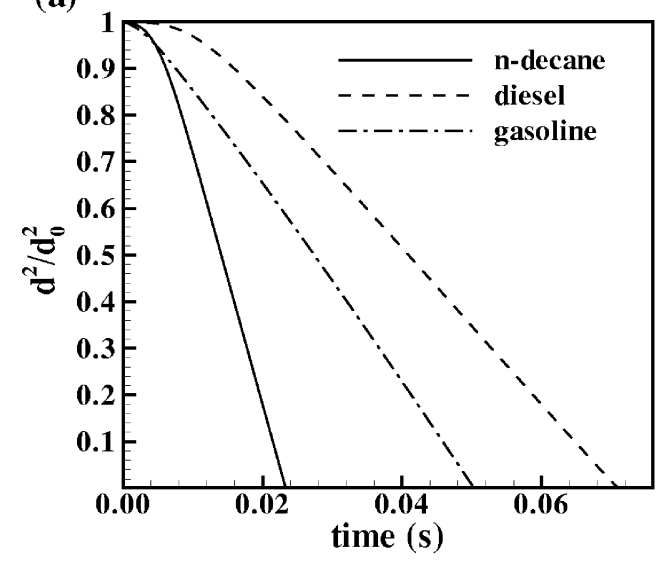

(c)

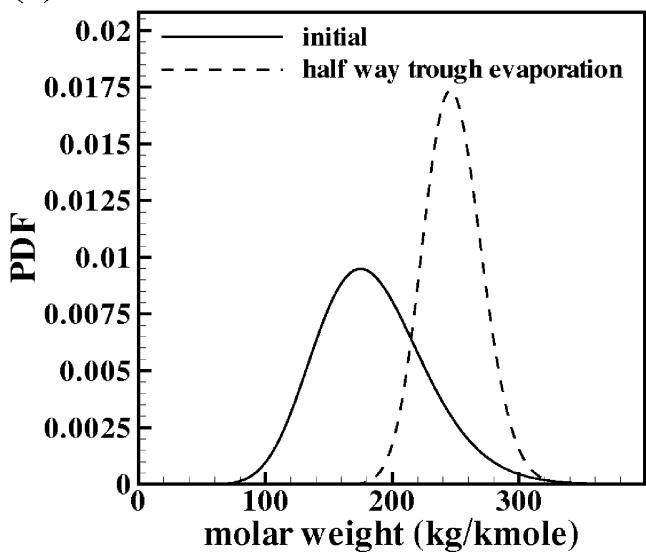

(b)

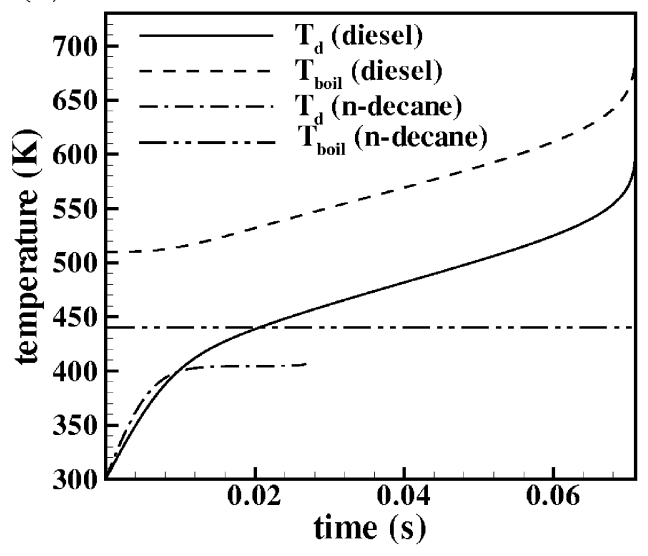

(d)

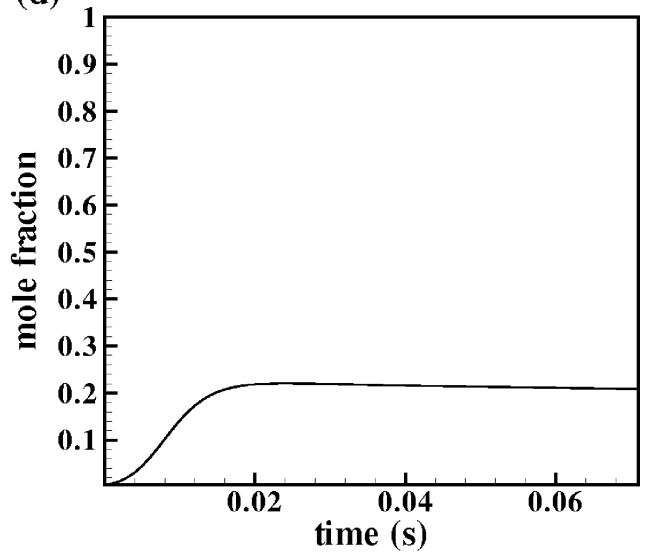

FIG. 2. Quiescent drop evaporation. Initial conditions: $T_{d, 0}=300 \mathrm{~K}, T_{e}=1000 \mathrm{~K}$, and $d_{0}=100 \mu \mathrm{m}$. (a) Normalized surface area, (b) drop and liquid boiling temperature evolution, (c) diesel-fuel drop composition evolution, and (d) surface mole fraction during diesel-fuel drop evaporation.

Rogers ${ }^{35}$ and Miller and Bellan. ${ }^{11,12} \delta_{\omega, 0}=\Delta U_{0} /\left\langle\partial u_{1} / \partial x_{2}\right\rangle$ where the brackets \langle\rangle indicate averaging over homogeneous $\left(x_{1}, x_{3}\right)$ planes, and the initial condition for $u_{1}$ is detailed in Ref. 11; for this initial condition $\delta_{\omega, 0}=6.85 \times 10^{-3} \mathrm{~m}$. The drops were distributed randomly throughout the $x_{2}<0$ domain with uniform number density and a uniform temperature $T_{d, 0}$. Table II summarizes the initial conditions, where the SC mixing layer results represent the database originated in Ref. 13. The initial drop slip velocity with respect to the gas is null, and the initial drop-size distribution is polydisperse, being specified by the Stokes number, St $=\tau_{d} \Delta U_{0} / \delta_{\omega, 0}$; both $\left\{\left\{\mathrm{St}_{0}\right\}\right\}$, where $\{\{\}\}$ denotes the drop ensemble average, and $\mathrm{St}_{0, R M S}$ are specified. Owing to the larger $\rho_{l}$ at same initial St, the MC calculations are initialized with a larger number of drops $N_{d, 0}$ and a smaller $\left\{\left\{d_{0}\right\}\right\}$ than their SC counterpart. Comparing MC and SC fuel parameters, it is obvious that one cannot have the same $\rho_{l}$, $\left\{\left\{d_{0}\right\}\right\}$, and $\mathrm{St}_{0}$. The choice of the same $\mathrm{St}_{0}$ in all computations means that the initial interaction of the drops with the flow is the same, and therefore if differences in the flow evolution occur, they are entirely the result of the SC versus $\mathrm{MC}$ aspect. For all simulations, the initial mass fraction of the evaporated species was null and in the MC simulations the initial molar weight was $137 \mathrm{~kg} / \mathrm{kmole}$ in the entire domain. $\mathrm{Re}_{0}$ was chosen small enough to obtain resolution of all scales. The mass loading $M L$ is defined as the total mass of the liquid relative to the total mass of the gas in the laden stream. Furthermore, in all simulations $M_{c, 0}=0.35$ and $\operatorname{Pr}$ $=\mathrm{Sc}=0.67$.

The numerical grid used in the simulations was uniform in all directions and is listed in the caption of Table II. The boundary conditions in the $x_{1}$ and $x_{3}$ directions were periodic and the adiabatic slip-wall conditions in the $x_{2}$ direction previously derived by Poinsot and Lele ${ }^{36}$ and Baum et al. ${ }^{37}$ based on the wave decomposition method, were here adapted to the CT model for MC mixtures (see the Appendix). Drops arriving at the slip-wall boundary were assumed to stick to it and were retained in the simulation, thus conserving mass. All simulations were performed on a SGI Origin 2000 platform, using 64 processors. The CPU time is listed in Table II and refers to the total for the processors.

The governing equations were solved numerically using a fourth-order explicit Runge-Kutta temporal integration for all time derivatives and eighth-order central finite differences for all spatial derivatives. A fourth-order Lagrange interpolation procedure was used to obtain gas-phase variable values at drop locations. As drops evaporate, their residual mass decreases. Drops whose residual mass was less than $3 \%$ were removed from the calculation. The initial conditions for the temporally developing mixing layer configuration were based on those of Ref. 35 and were detailed in Ref. 11.

One computational difficulty in mixing-layer MC-fuel 


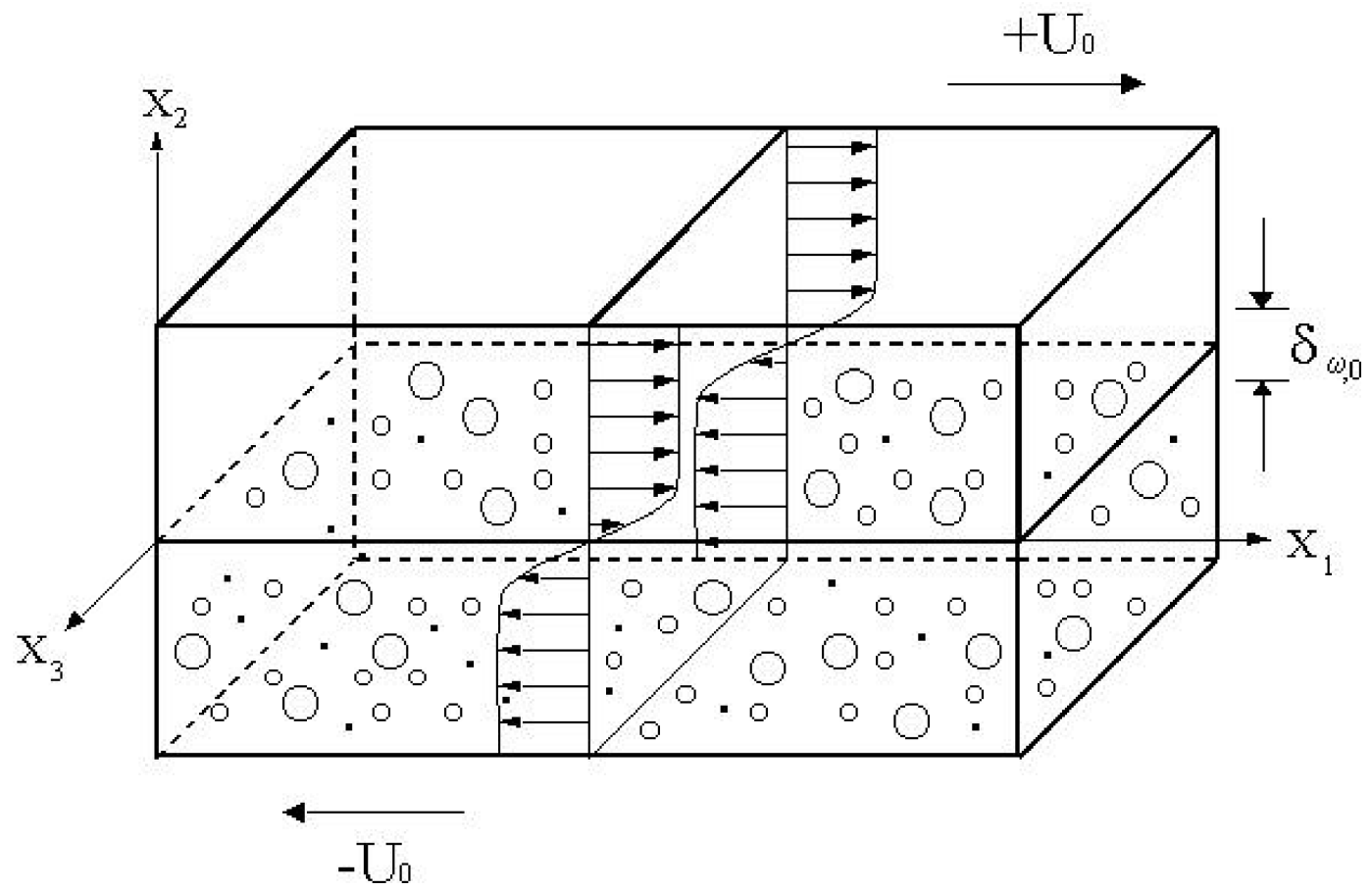

FIG. 3. Mixing layer configuration.

drop simulations is determining $K$ and $K^{\prime}$ in the CT form of $h_{v}^{0}(m), h_{v}^{0}\left(\theta_{v}\right)=\int_{0}^{\infty} h_{v}^{0}(m) f_{v}(m) d m=K \theta_{v}+K^{\prime}$. For an isolated MC-fuel drop whose far-field conditions are specified, Eq. (25) becomes

$$
\begin{aligned}
L_{v}\left(T_{u \cdot b}\right)= & \underbrace{K \frac{X_{v s} \theta_{v s}(1+B)-X_{v e} \theta_{v e}}{B}+K^{\prime}}_{\text {reference enthalpy } h_{v}^{0}} \\
& +C_{l} T_{u^{\prime} b}-R_{u} T_{u^{\prime} b} A_{c}-R T_{u^{\prime} b} B_{c} \\
& \times \frac{X_{v s} \theta_{v s}(1+B)-X_{v e} \theta_{v e}}{B} .
\end{aligned}
$$

Therefore $K^{\prime}$ can be obtained from Eq. (64) at the initial condition, and $K$ can be found as a function of $\theta_{v s}$. Although in principle the same procedure can be applied for the mixing layer drops whose far-field conditions change as a function of position and time, this calculation introduces a large computational overhead. To remove this computational overhead, single-drop computations were performed for diesel fuel at several initial conditions, and the values of $K$ and $K^{\prime}$ were empirically determined (as the composition of the drop changed with time) from a plot of $h_{v}^{0}$ vs $\theta_{v s}$ created according to Eq. (64). While it is apparent that $h_{v}^{0}$ is not strictly linear and depends on the initial conditions, for ease of computation in the mixing layer simulations, the linear relationship for $h_{v}^{0}$ was retained, with $K=-100175 \mathrm{~J} / \mathrm{kg}, K^{\prime}$ $=119663800 \mathrm{~J} / \mathrm{kmole}$. Although the linear relationship is only qualitatively correct, it embodies the general behavior exhibited in these calculations and it is thus deemed appropriate considering the uncertainty associated with the thermophysical parameters for MC-fuel distributions. All transport properties were calculated at $350 \mathrm{~K}$.

\section{B. Global layer evolution and transition attainment}

To compare SC and MC simulations, the global evolution of the layers through the momentum thickness $\delta_{m}$,

$$
\delta_{m}=\frac{1}{\left(\theta_{1}-\theta_{2}\right)^{2}} \int_{L_{2, \text { min }}}^{L_{2, \max }}\left(\theta_{2}-\left\langle\rho u_{1}\right\rangle\right)\left(\theta_{1}-\left\langle\rho u_{1}\right\rangle\right) d x_{2},
$$

TABLE II. Simulation parameters. For all simulations $M_{c, 0}=0.35, \mathrm{Re}_{0}=500, T_{d, 0}=345 \mathrm{~K}$, and $T_{0}=375 \mathrm{~K}$, and for MC simulations $\gamma_{l, 0}=76 \mathrm{~kg} / \mathrm{kmole}$. The initial drop size distribution is polydisperse and Gaussian with $\left\{\left\{S t_{0}\right\}\right\}=3$ and $\left\{\left\{\left(S t_{0}-\left\{\left\{S t_{0}\right\}\right\}\right)^{2}\right\}\right\}^{1 / 2}=0.5$. The resolution was $N_{1} \times N_{2} \times N_{3}=256 \times 288 \times 160$ in all simulations. SC2 and SC5 represent databases obtained in Okong'o and Bellan (Ref. 13).

\begin{tabular}{lccccccc}
\hline \hline & & & & & \multicolumn{2}{c}{$t^{*}=t \Delta U_{0} / \delta_{\omega, 0}$} & \\
\cline { 5 - 6 } Run & Fuel & $M L_{0}$ & $N_{d, 0}$ & $\left\{\left\{d_{0}\right\}\right\}, \mathrm{m}$ & $\begin{array}{c}\text { Peak in } \\
\left.\left\langle\omega_{3}^{+}\right\rangle\right\rangle \delta_{\omega, 0} / \Delta U_{0}\end{array}$ & Transition & $\begin{array}{c}\text { CPU time (at } \\
\text { transition), hours }\end{array}$ \\
\hline SC2 & $n$-decane & 0.2 & $2.28 \times 10^{6}$ & $8.6 \times 10^{-5}$ & 86 & 100 & 1336 \\
SC5 & $n$-decane & 0.5 & $5.70 \times 10^{6}$ & $8.6 \times 10^{-5}$ & 97 & 105 & 2075 \\
MC2 & diesel & 0.2 & $2.70 \times 10^{6}$ & $7.6 \times 10^{-5}$ & 98 & 105 & 2340 \\
MC5 & diesel & 0.5 & $6.50 \times 10^{6}$ & $7.6 \times 10^{-5}$ & 103 & 110 & 4256 \\
\hline \hline
\end{tabular}


a)

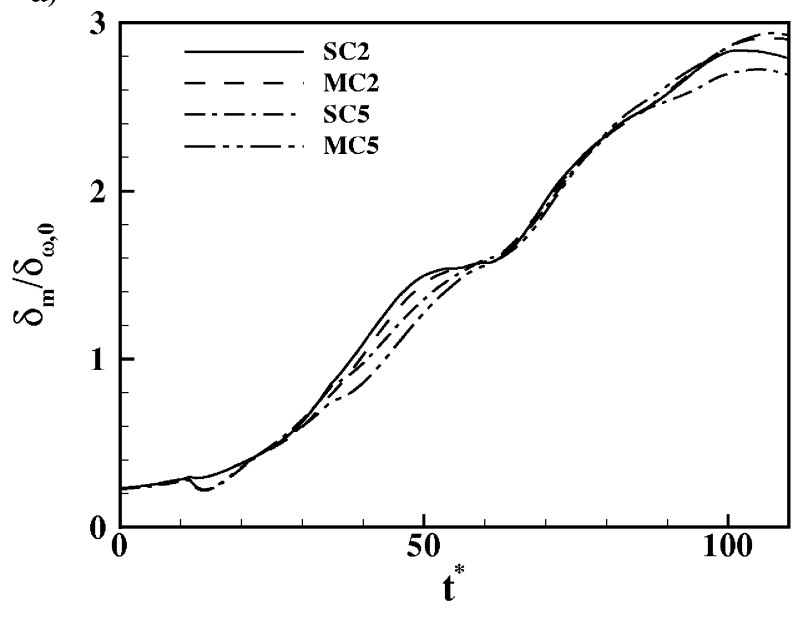

b)

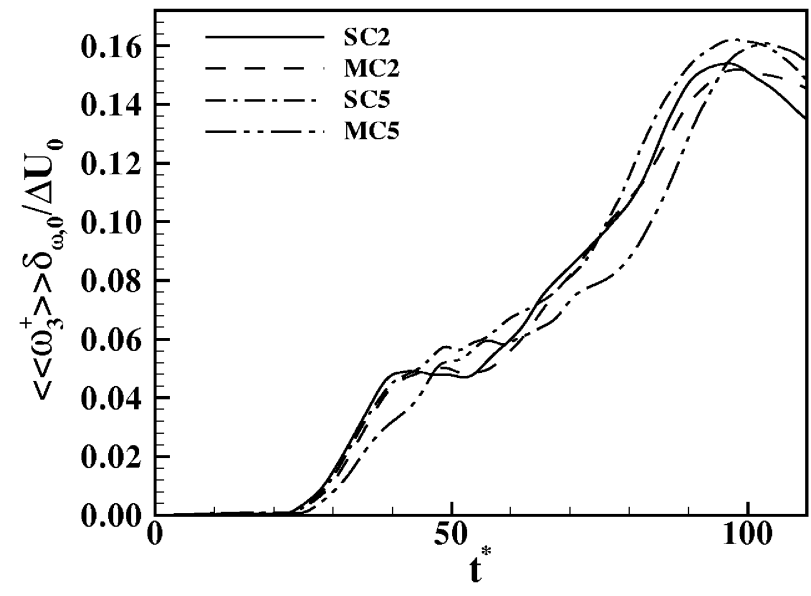

c)

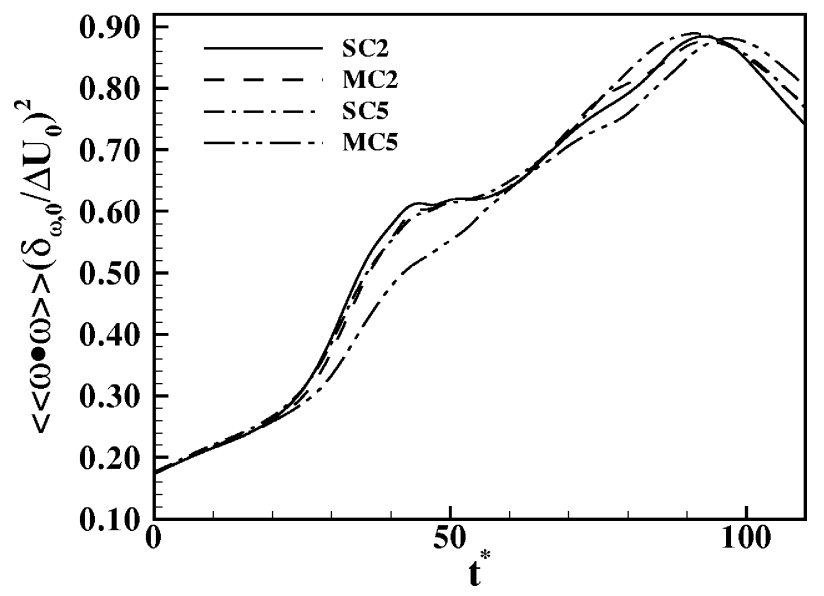

FIG. 4. Timewise evolution of the nondimensional momentum thickness, positive spanwise vorticity and enstrophy for the simulations listed in Table II.

was first examined, with $\theta_{1}=\left\langle\rho u_{1}\right\rangle_{x_{2}=L_{2, \max }}$ and $\theta_{2}$ $=\left\langle\rho u_{1}\right\rangle_{x_{2}=-L_{2, \min }}, L_{2, \max }=L_{2} / 2$ and $L_{2, \min }=-L_{2} / 2$. Illustrated in Fig. 4(a) is $\delta_{m} / \delta_{\omega, 0}$ as a function of $t^{*}$ $=t \Delta U_{0} / \delta_{\omega, 0}$ for both SC-fuel and MC-fuel drop-laden layers. All layers display rollup and a double pairing, with a plateau after the first pairing indicative of the forcing effect. At same $M L_{0}$ one discerns little difference between the SC and MC

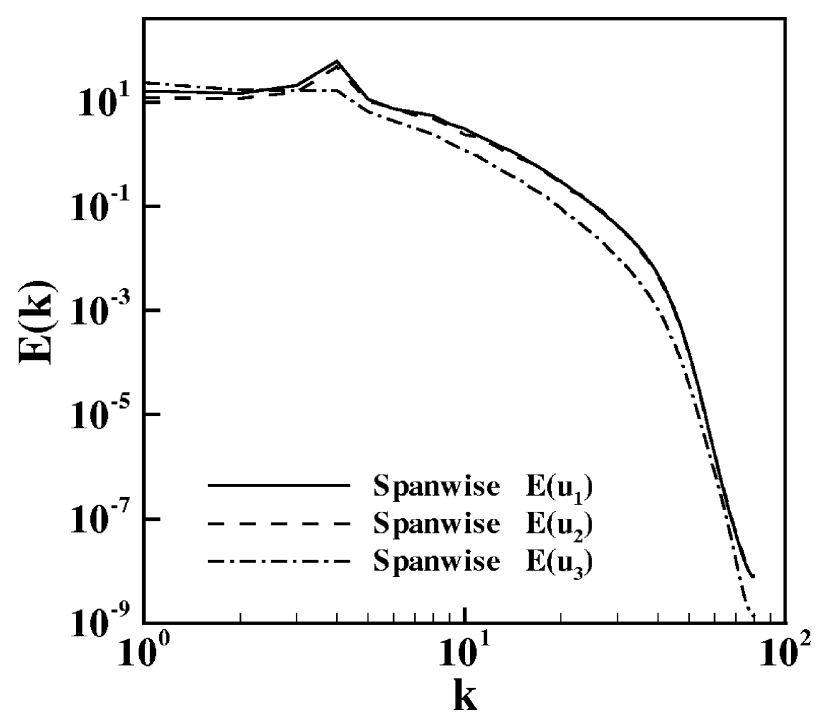

FIG. 5. Spanwise energy spectra for each component of the velocity fluctuations for MC5 at $t^{*}=110$.

layer growth, with the SC layers growing somewhat larger before the first pairing and vice versa after the second pairing. The larger $M L_{0}$ layers exhibit a smaller and more linear growth with lesser influence from the forcing, this being attributed to the higher density stratification between the two streams resulting in more difficulty to entrain. The SC5 layer displays the smallest ultimate growth, with the smallest momentum-thickness-based Reynolds number, $\operatorname{Re}_{m}$ $\equiv \operatorname{Re}_{0} \delta_{m} / \delta_{\omega, 0}$, at transition (1415 for SC2, 1450 for MC2, 1360 for SC5, and 1465 for MC5). The larger $\mathrm{Re}_{m}$ at transition for MC-fuel simulations compared to their SC counterpart indicates that these former layers may have enhanced turbulent features compared to the latter.

Owing to the major role of vorticity, $\omega \equiv \nabla \times \mathbf{u}$, in turbulent flows, global aspects of the flow that are associated with $\omega$ were also examined. In Figs. 4(b) and 4(c) the volume averaged nondimensional positive spanwise vorticity, $\left\langle\left\langle\omega_{3}^{+}\right\rangle\right\rangle \delta_{\omega, 0} / \Delta U_{0}$, and the nondimensional enstrophy, $\left\langle\left\langle\omega_{i} \omega_{i}\right\rangle\right\rangle\left(\delta_{\omega, 0} / \Delta U_{0}\right)^{2}$, evolutions are depicted as functions of $t^{*}$ where $\langle\langle\rangle\rangle$ denotes volume averaging. The positive spanwise vorticity [Fig. 4(b)] begins increasing after rollup, and following the plateau displayed after the first pairing continues to increase at a sustained rate. Although the SC5 layer initially follows the growth of the $M L_{0}=0.2$ layers, it eventually reaches the highest peak, indicating that the increased number of drops contributes to an increased formation of small scales. With respect to the other three layers, the MC5 layer has a delayed growth and peak (peak times in Table II), which is, however, still larger than that of both $M L_{0}=0.2$ layers, albeit slightly smaller than that of the SC5 layer. The indications are that the MC layers achieve a slightly reduced small-scale formation than their SC counterpart. A similar behavior to that of $\omega_{3}^{+}$is portrayed in Fig. 4(c) for the enstrophy, but we note that these indications pertain only to the attained maxima and are reversed past the culminating point of the curves. With increasing $M L_{0}$, the difference in enstrophy evolution between the SC and MC layers becomes enhanced, with the MC5 layer evolving 
in a more linear manner, lagging in stretching and tilting activity, and displaying a delayed peak with respect to the other layers, although all layers reach similar enstrophy levels. To understand the relatively small sensitivity to the fuel composition, the $\omega$ and $\omega \cdot \omega$ equations were derived and their budget was evaluated (not shown). The largest contribution to vorticity production is from the stretching and tilting term followed by the viscous term, while the source terms have negligible contributions. Therefore the small sensitivity of the global vorticity aspects to the fuel composition may be traced to the negligible role of the source terms for these values of $M L_{0}$. However, one discerns a generally higher activity in the vorticity-magnitude rms and an enhanced effect of viscosity in the $\omega_{3}$ rms for the SC-fuel drop-laden layer.

By our stringent criterion of mixing transition achievement, not only must $\delta_{m} / \delta_{\omega, 0}, \quad\left\langle\left\langle\omega_{3}^{+}\right\rangle\right\rangle \delta_{\omega, 0} / \Delta U_{0}$ and $\left\langle\left\langle\omega_{i} \omega_{i}\right\rangle\right\rangle\left(\delta_{\omega, 0} / \Delta U_{0}\right)^{2}$ increase sharply and in a sustained manner, but also the spectra must be smooth, indicative of the full range of scales characteristic of turbulence. Illustrated as an example, Fig. 5 plots represent the spanwise spectra for the MC5 simulation at the time identified in Table II as corresponding to mixing transition. These plots display the full range of scales indicative of transition. The peak in the energy spectra at a wave number of 4 is attributed to the spanwise forcing. The plots additionally show that the flow is completely resolved, as most of the energy is in the low wave-number regime and there is no energy accumulation in the high wave numbers.

\section{Flow characteristics and drop organization at transition}

Since local conditions are of prime interest (e.g., govern reaction rates), it is pertinent to examine the local vorticity, drop number distribution and the evaporated-fuel mass fraction.

\section{Vorticity}

Comparison of contour plots of $\omega_{3}$ for SC2 and MC2 after the second pairing $\left(t^{*}=87\right)$ show a markedly different local structure (not shown). The maximum $\omega_{3}^{+}$is smaller for the MC-fuel drop-laden layer, indicating that a wide spread molar-weight liquid-fuel composition may globally impede vorticity production; this conclusion is consistent with the vorticity and vorticity-magnitude budget analyses. Although the general level of vorticity activity is higher in the SC-fuel layer, locally one observes considerably more numerous high vorticity regions for the $\mathrm{MC}$-fuel mixing layer. These sites of very high vorticity lead to a more highly structured flow (not shown) by inducing the formation of high drop number density regions through flinging the drops away from the high to the low vorticity locations. Since transition is attained following the $\omega_{3}^{+}$peak, the vorticity level is reduced from that at $t^{*}=87$, however, the SC-fuel layers retain larger $\omega_{3}^{+}$with respect to the MC-fuel layers. Figure 6 shows $\omega_{3}$ in the between-the-braid plane for the SC [Figs. 6(a) and 6(b)] and MC [Figs. 6(c) and 6(d)] layers at transition; the braid plane plots display a similar behavior. Numerous sites of positive spanwise vorticity, plotted in solid lines, are obvious in all figures, indicating small-scale production. Although the major visual features of the flow are similar for SC2 and MC2 simulations, the details are different. Noteworthy, the MC2 layer exhibits a spottier aspect with isolated localities of high vorticity. As $M L_{0}$ increases, the ratio of the maximum positive spanwise vorticity level between SC and MC simulations becomes larger and it becomes easier to discern the more numerous regions of high vorticity for the MC-fuel layer.

Because SC layers have regions of larger vorticity magnitude at transition, these results do not support the global analysis indication that $\mathrm{MC}$ layers may have more turbulence activity than SC ones. However, MC layers display more small-scale structure than their SC equivalent, an aspect confirmed by the drop organization examined below.

\section{Drop organization}

Since MC calculations are initiated with larger $N_{d}$ than their SC equivalent, the pertinent quantity to examine is not $N_{d}$ but the drop-number density $\rho_{n}$. Visualizations of $\rho_{n}$, calculated as an Eulerian field from the instantaneous Lagrangian droplet locations,

$$
\rho_{n}=\sum_{q=1}^{N_{d}} \frac{w_{q}}{\Delta x^{3}},
$$

show the relationship between the regions of high vorticity and relatively small $\rho_{n}$. The results presented in Figs. 7(a)7 (d) are at transition and qualitatively agree with those of Squires and Eaton, ${ }^{38}$ who found that particles with a density larger than that of the carrier flow concentrate in regions of low vorticity and high strain. They are also reminiscent of the "focusing" effect defined by Crowe et al. ${ }^{39}$ for coherent vortices in the laminar flow context; however, in Fig. 7 the noteworthy feature is the small-scale structure formed, with drops profiling the small-scale instead of the coherent vortices. The highest drop concentration $\left(3.43 \times 10^{10}\right.$ for $\mathrm{SC} 2$, $3.16 \times 10^{10}$ for MC2, $4.6 \times 10^{10}$ for SC5, and $6.0 \times 10^{10}$ for MC5, all in $\mathrm{m}^{-3}$; these maxima are not necessarily shown on Fig. 7 because they occur at pointwise locations and cannot be captured by a color rendering scale representative of the important features) is not at the periphery of the coherent vortex representing the ultimate structure resulting from the two pairings of the four initial vortices, but rather at the periphery of small-scale vortical structures. Following the second pairing, at $t^{*}=87, \rho_{n}$ exhibits considerably larger levels and displays much more structure for the MC2 than for the SC2 simulation (not shown). The smaller $\rho_{n}$ is attributed to the faster drop evaporation (see Sec. IV D 1) inducing some drops to be completely evaporated and thus removed from the computation, whereas none of the drops in the MC2 simulation satisfy the criterion for complete evaporation; the fact that some of the SC drops have evaporated while none of the MC drops did so is significant, as $\left\{\left\{d_{0}\right\}\right\}$ was slightly larger for the SC simulations. The larger $\rho_{n}$ may also be attributable to the larger $N_{d, 0}$ in $\mathrm{MC}$ computations. Comparing $\rho_{n}$ for the $\mathrm{SC} 2$ and $\mathrm{MC} 2$ simulations at transition [Figs. 7(a) and 7(c)], it appears that this evidence of higher maximum $\rho_{n}$ for the MC2 simulation accompanied by an in- 

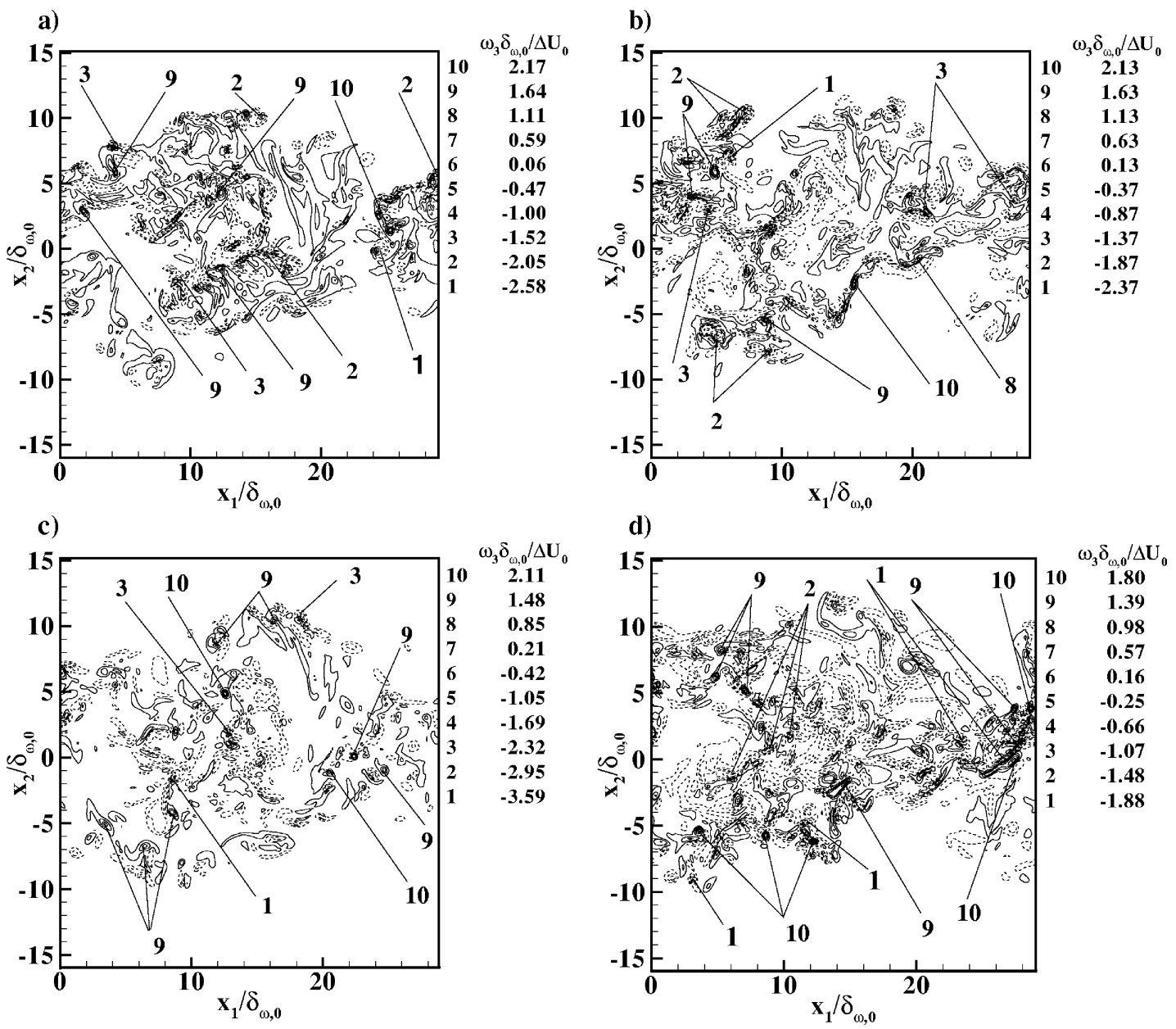

FIG. 6. Nondimensional spanwise vorticity in the between-the-braid plane $\left(x_{3}=0.06 \mathrm{~m}\right)$ at the transitional time for (a) SC2, (b) SC5, (c) MC2, and (d) MC5.

creased small-scale structure is lost, indicating drop reorganization. Except for more numerous locations of high $\rho_{n}$ indicative of an increased structure for the MC5 compared to the SC5 simulation [compare Figs. 7(b) and 7(d)], it is similarly difficult to quantify from simple visual inspection the differences between the two sets of results. The molar weight distribution depicted in Figs. 7(e) and 7(f) is illustrated here for the purpose of direct visual comparisons with Figs. 7(c) and 7(d) and is discussed in detail in Sec. IV C 3.

To quantify the drop structure in the flow, we calculated the drop-number density conditionally averaged on the second invariant of the deformation tensor for compressible flow,

$$
I I_{u}=-\frac{1}{2}\left[\left(S_{i j} S_{i j}-S_{k k} S_{l l}\right)-\frac{1}{2} \omega_{i} \omega_{i}\right],
$$

and on a passive scalar $\phi$ (here the fuel mass fraction) which is chosen as $\phi<0.5$ to ensure that primarily only ladenstream fluid is considered. The form of $I I_{u}$ is conducive to distinguishing portions of the flow that are of rotational or compressible nature, corresponding to $I I_{u}>0$, from other portions of the flow where strain dominate, corresponding to $I I_{u}<0$. Noteworthy, because the rotational part is weighted by $1 / 4$ whereas the strain/compressibility part is weighted by $1 / 2$, conditioning on $I I_{u}$ does not give an accurate portrayal of the relative $\rho_{n}$ in these regions; to obtain an accurate proportion one would have to weigh appropriately the positive and negative contributions. However, this subtlety does not intervene if the intent is to compare the results from different simulations. Displayed in Fig. 8 are the nondimensional conditional averages of $\rho_{n}$ for all simulations listed in Table II. Even with the uncertainties about comparing the $\rho_{n}$ magnitude for positive and negative contributions, it is obvious that most drops accumulate predominantly in regions where $I I_{u} \simeq 0$, then in regions where $I I_{u}<0$ and finally at locations where $I I_{u}>0$. On the scale of the plot, in the moderate and strongly positive $I I_{u}$ regions, $\rho_{n}$ is merely a function of $M L_{0}$, with only subtle differences in regions of low vorticity and compressibility, where the SC simulations exhibit slightly larger values than their MC counterpart. However, larger differences between SC and MC results appear in the $I I_{u}<0$ regions. For $M L_{0}=0.2$, the plots in Fig. 8 show that with the exception of very strongly negative $I I_{u}$ regions, $\rho_{n}$ is larger for MC2 than for SC2 in strain regions, indicating that $\rho_{n}$ remains larger for MC2 past the second pairing and at transition. For $M L_{0}=0.5$, the results exhibit an even larger difference between the SC versus MC simulations, and in very strong strain regions $\rho_{n}$ is considerably larger for MC5 than for SC5. The highly nonmonotonic variation of the MC plots in moderately to strongly $I I_{u}<0$ regions indi- 
a)

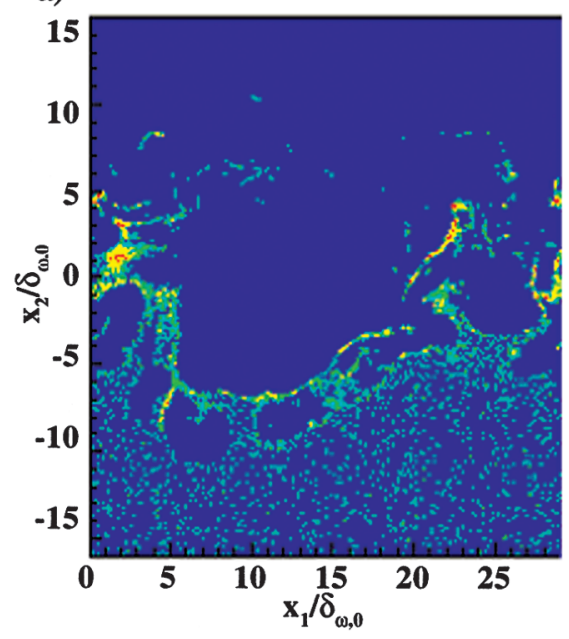

c)

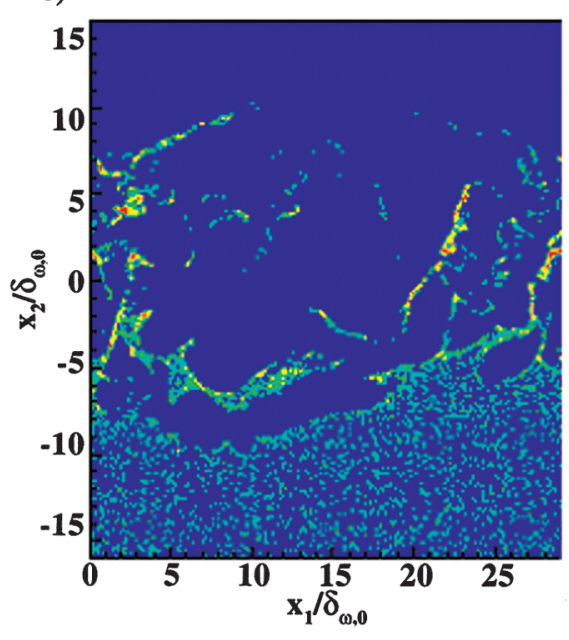

e)

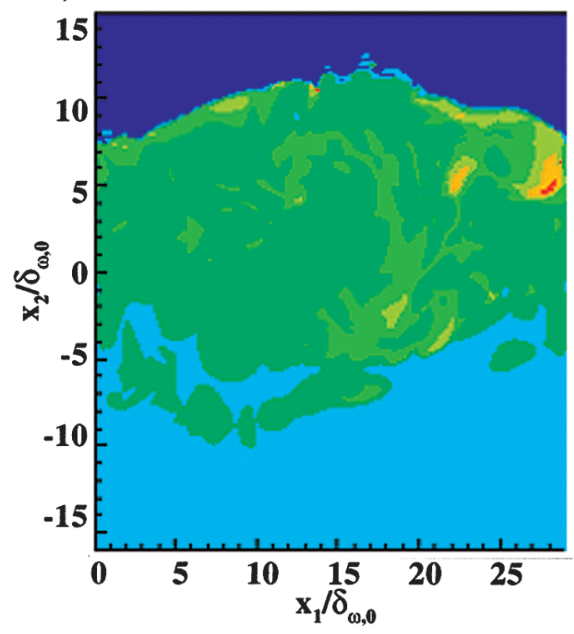

b)
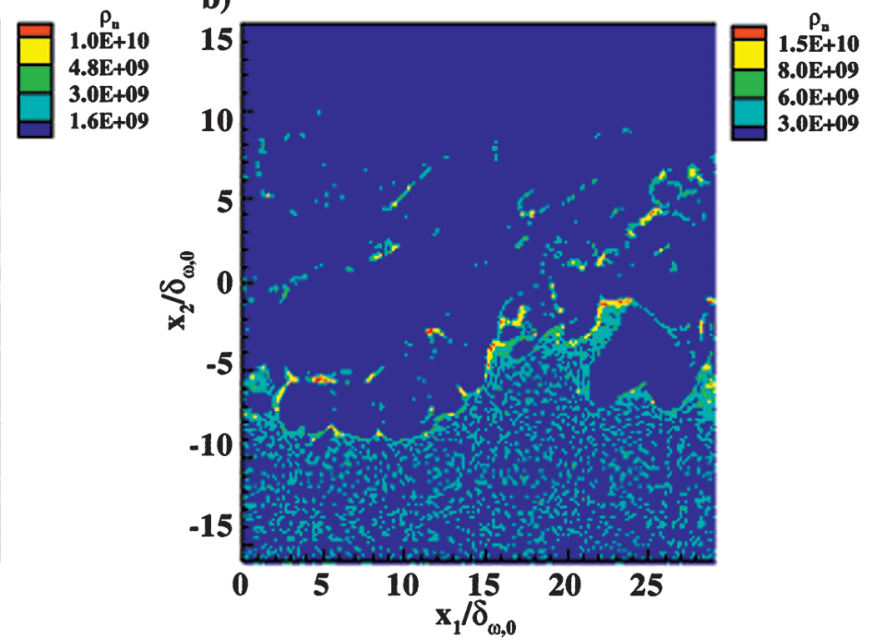

d)
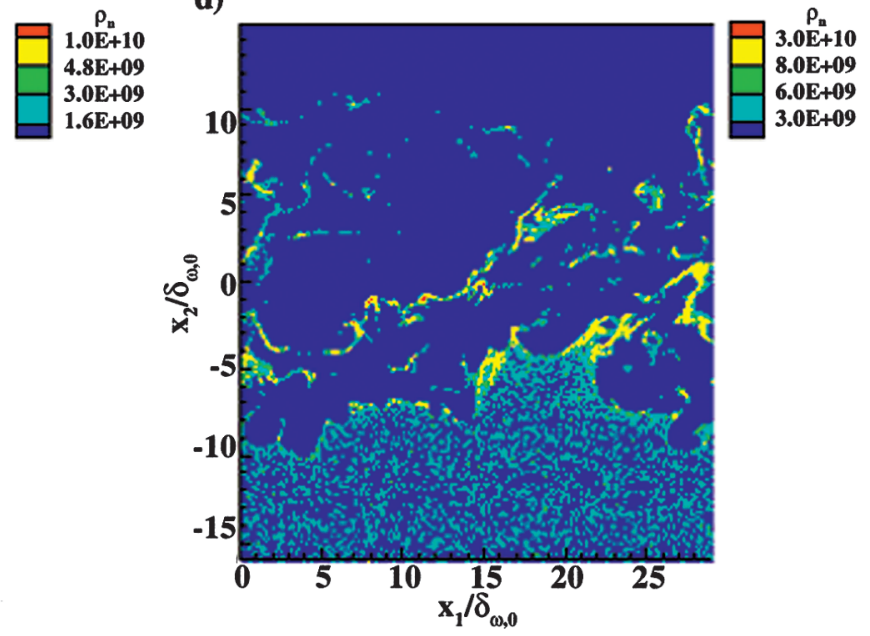

f)
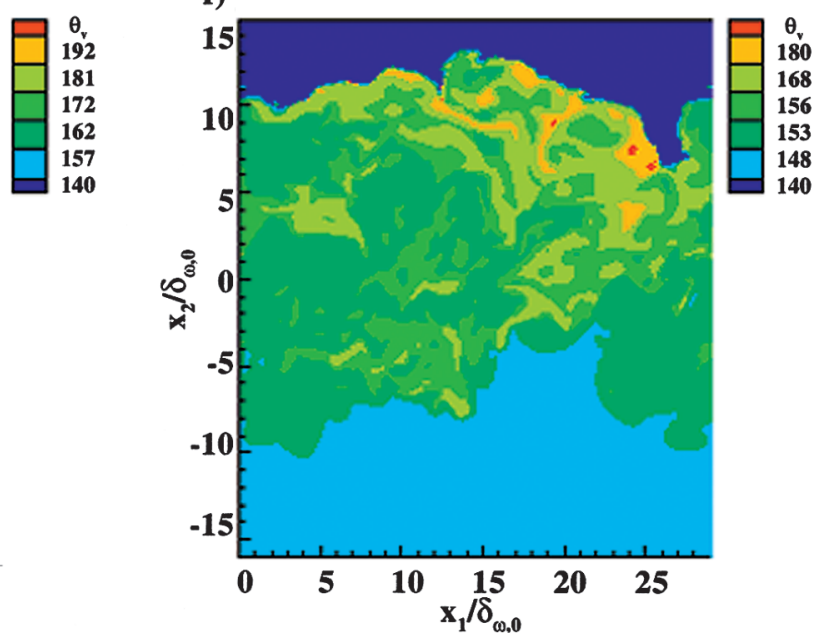

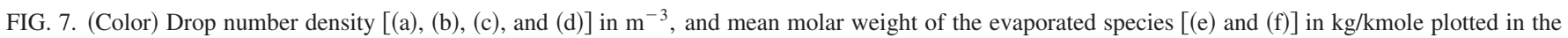
between-the-braid plane $\left(x_{3}=0.06 \mathrm{~m}\right.$ ) at the transitional time for (a) SC2, (b) SC5, (c) MC2, (d) MC5, (e) MC2, and (f) MC5.

cates an increased small-scale structure with respect to their $\mathrm{SC}$ equivalent. These quantitative results confirm the qualitative observations from Figs. 7(b) and 7(d) and show that the MC-liquid composition impacts the $\rho_{n}$ magnitude and its distribution. The reasons for this occurrence are discussed in Sec. IV D.

\section{Molar weight distribution for MC-fuel simulations}

The distribution of gas-phase molar weight at transition is shown in Figs. 7(e) for MC2 and Fig. 7(f) for MC5 in the between-the-braid plane. Because of color rendering, the full range of molar weights cannot be displayed (the maximum 


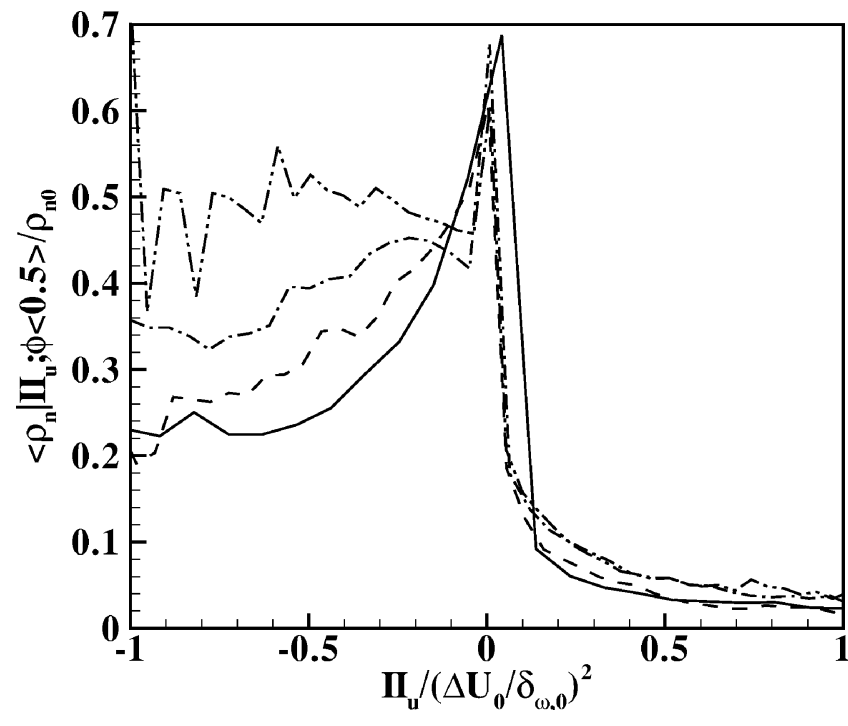

FIG. 8. Drop number density at the transitional times conditioned on the second invariant and on a passive scalar being smaller than 0.5. - SC02, - - MC02, -... SC05, -...... MC05.

molar weight was $250 \mathrm{~kg} / \mathrm{kmole}$ for MC2 and $212 \mathrm{~kg} / \mathrm{kmole}$ for MC5), however, the deleted values in the upper range of molar weights occupy only point-like locations; for each of the two figures, the maximum shown molar weight was chosen based on the best color representation for the entire domain. The larger maximum $\theta_{v}$ obtained in the MC2 simulation is attributed to the larger $T_{d}$ (the ensemble averaged $T_{d}$ is discussed in Sec. IV D 2), which allows the evaporation of less volatile species whose molar weight is larger. Despite removing the largest values from the illustrations, the wide range of values and the very complex molar weight local distribution is noteworthy, to be compared to the constant molar weight of $n$-decane, $142 \mathrm{~kg} / \mathrm{kmole}$, which is spatially distributed in the domain according to $Y_{v}$ (see below). Most of the lighter components, which egress from the drop earlier during evaporation, reside in the lower stream. The intermediary-weight components, which are released after the drops have been entrained, reside mostly in the interior of the layer as they have already participated in the mixing process. In contrast, the heavier components reside in the high $\rho_{n}$ regions, as they evaporate later during the drop lifetime and have not participated in the mixing; in chemically reacting flows, heavier species will lead to solid-particulate formation, an undesirable aspect of combustion. Therefore a segregation of chemical species becomes established according to their release time from the drops. This segregation inherently cannot be captured by SC-fuel simulations.

In the between-the-braid plane, the MC5 layer encompasses more numerous high-molar-weight locations than the MC2 layer, consistent with the more numerous high $\rho_{n}$ locations in the layer and with the larger maximum $\rho_{n}$. Also, the molar weight distribution is more inhomogeneous with increasing $M L_{0}$. In order to quantify the molar weight distribution across the layer at transition, $\left(x_{1}, x_{3}\right)$-plane average $\theta_{v}$ PDFs of the molar weight that are illustrative of the lower stream $\left(x_{2} / \delta_{\omega, 0}=-12\right)$, lower part of the layer $\left(x_{2} / \delta_{\omega, 0}\right.$ $=-2.5)$, and upper part of the layer $\left(x_{2} / \delta_{\omega, 0}=7\right)$ were cal-

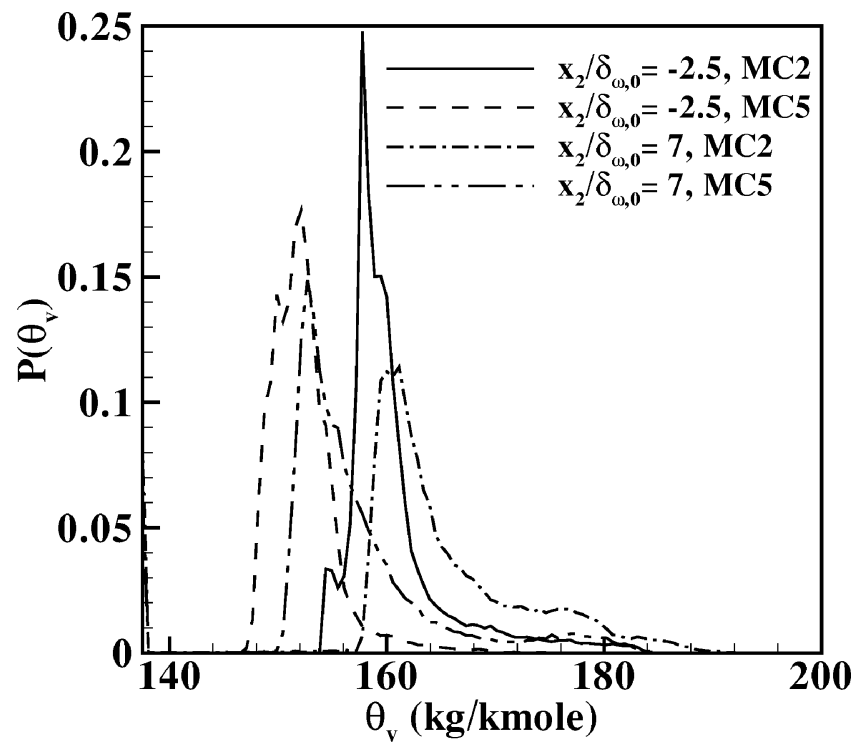

FIG. 9. PDF of $\theta_{v}$ at different cross-stream locations, $x_{2} / \delta_{\omega, 0}=-2.5$ and 7 for both MC2 and MC5 layers.

culated. The PDF in the lower stream peaks at lower $\theta_{v}$ values and is akin to a delta function (not shown) culminating at lower $\theta_{v}$ values with increasing $M L_{0}(156 \mathrm{~kg} / \mathrm{kmole}$ for MC2 and $147 \mathrm{~kg} / \mathrm{kmole}$ for MC5). The PDFs in the lower and upper parts of the layer are illustrated in Fig. 9. In the lower layer region the PDFs peak at higher values than in the lower stream and the peak location remains at smaller values with increasing $M L_{0}$. The small "bumps" on each side of the peak correspond to regions of high $\rho_{n}$ where high-molarweight components reside. In the upper part of the layer, the PDFs widen in the higher $\theta_{v}$ range and their peak value decreases, with the higher peak now corresponding to the larger $M L_{0}$. The wider range is illustrative of the higher molar weight of components evaporated late in the drop lifetime and residing in the upper layer high- $\rho_{n}$ region [e.g., Figs. 7(e) and 7(d)]. The small local peak evident in the low $\theta_{v}$ range represents a region of small molar weights traversed by the $\left(x_{1}, x_{3}\right)$ plane at $x_{2} / \delta_{\omega, 0}=7$ (e.g., Fig. 7).

Results showing segregation of species have been found in laminar combustion simulations by Continillo and Sirignano $^{40}$ who used a binary-fuel species as a representation for diesel or kerosene and a detailed transport model in conjunction with a drop field representation by groups of drops (the number of groups seems unspecified) rather than the present physical drops; the segregation was attributed to the different volatilities of the components. The present results show that this segregation also occurs for MC fuels in transitional flows which indicates that turbulence may be ineffective in homogenizing the flow composition.

\section{Evaporated-fuel mass fraction}

The mass fraction of the evaporated species is illustrated in Fig. 10. Comparing the results from SC and MC simulations, it is apparent that the drops in the latter produce a smaller amount of vapor (see legends; the maxima, 0.096 for SC2, 0.1 for SC5, 0.067 for MC2, and 0.078 for MC5, are not displayed), which is attributed to the wide range of spe- 
a)

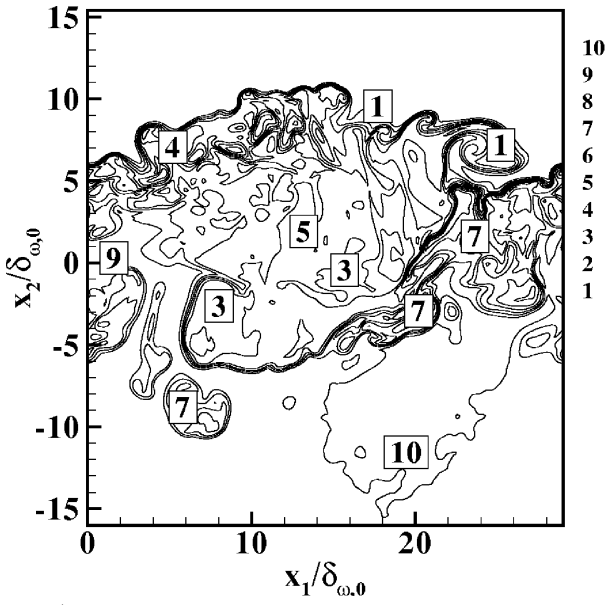

c)

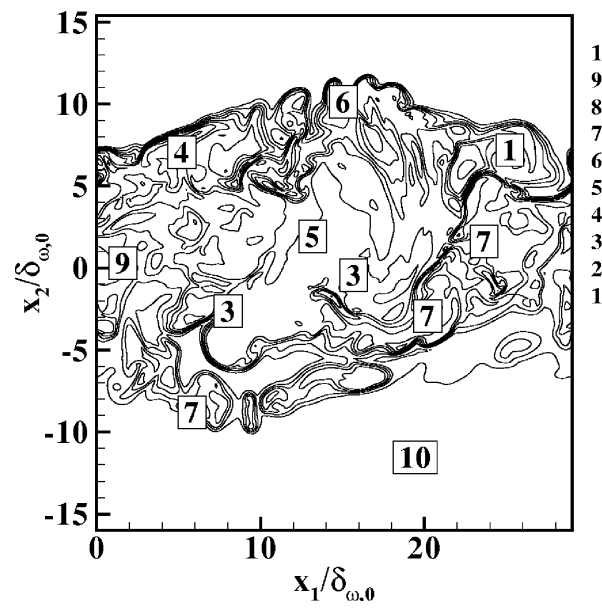

b)
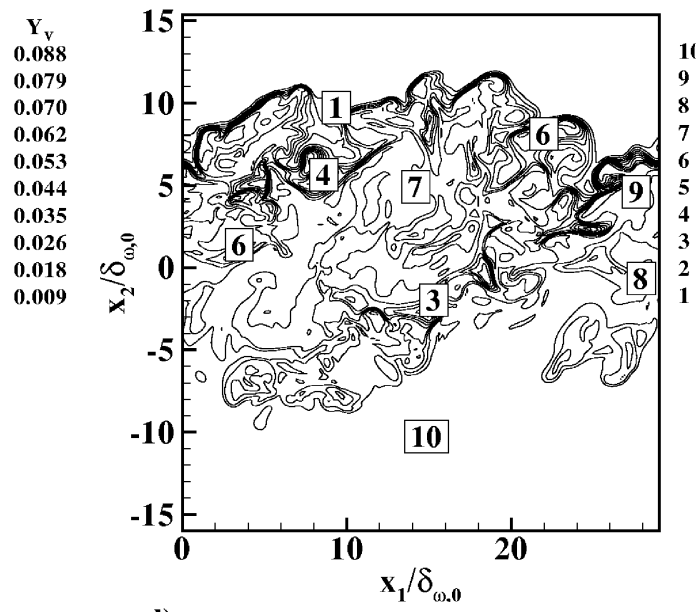

$\mathrm{Y}_{\mathrm{V}}$

$\mathbf{0 . 0 9 3}$

0.074

0.065

0.056

0.046

$\mathbf{0 . 0 3 7}$

0.028

0.019
0.009

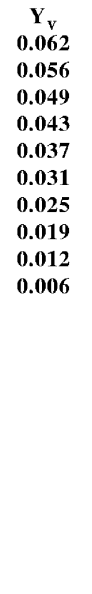

d)

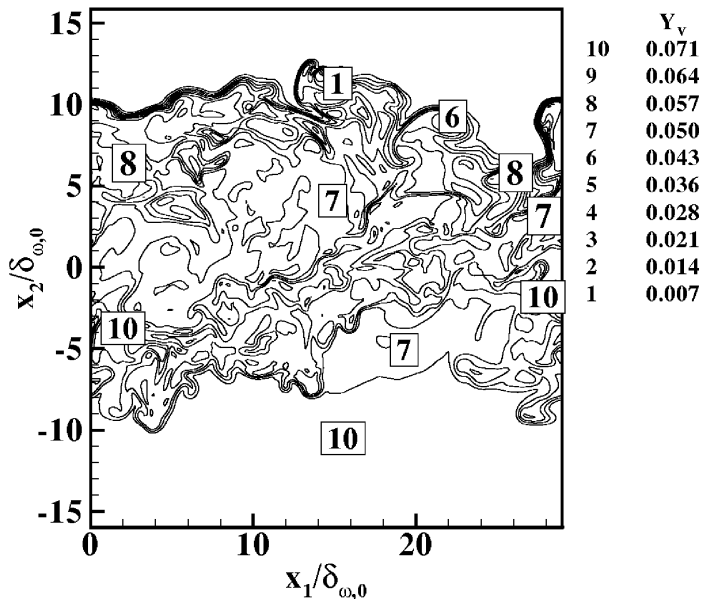

FIG. 10. Mass fraction of the evaporated species in the between-the-braid plane ( $x_{3}=0.06 \mathrm{~m}$ ) at the transitional time for (a) SC2, (b) SC5, (c) MC2, and (d) MC5.

cies volatility that leads to a decreasing evaporation rate once the most volatile components have been released. This behavior has already been identified in the single-drop simulations discussed in Sec. III. Regions of larger $Y_{V}$ generally correspond to the locations of high $\rho_{n}$, however, moderate $Y_{V}$ regions occur throughout the layer owing to gas-phase transport mechanisms carrying evaporated species from the drop surface to other layer regions.

\section{First-order statistics}

The first-order statistics are calculated to enable a more complete understanding of the global and detailed features of the layers. For the drop variables, these statistics are Lagrangian, meaning that averages are performed on the drop ensemble. The gas-phase variables are subjected to volumetric Eulerian averages.

\section{Drop size}

Illustrated in Fig. 11(a) is the Lagrangian ensemble average of the residual drop diameter in the entire domain as a function of $t^{*}$; comparing nondimensional rather than dimensional drop diameters removes the bias introduced by the smaller $\left\{\left\{d_{0}\right\}\right\}$ in MC simulations. The linear behavior of the well-known $d^{2}$ law is not observed in Fig. 11 as the curves represent ensemble averages rather than the individual drop behavior. To understand the different fate of drops in the mixing layer (defined as $x_{2} / \delta_{\omega, 0} \geqslant-7$ ) from those in the lower stream (i.e., $x_{2} / \delta_{\omega, 0}<-7$ ), separate Lagrangian ensemble averages are depicted in Fig. 11(b). For $M L_{0}=0.2$, the MC-fuel drops evaporate considerably slower than the SC-fuel drops [Fig. 11(a)] due to the decreased volatility accompanying the increase in molar weight. This decreased evaporation induces the drops to have more interaction with the flow, and this increased coupling results in the augmented small-scale structure discussed in Sec. IV C 2. The evaporation rate is considerably reduced when $M L_{0}=0.5$. Also, although the MC5 evaporation rate is slower than in SC5, the difference between the two simulations is considerably smaller than when $M L_{0}=0.2$. As stated earlier, the smaller evaporation rate of the MC simulations is attributed to the wide range of volatilities that reduces evaporation once the most volatile components have been released; the smaller evaporation rate for $M L_{0}=0.5$ simulations is attributed to the larger number of drops, which represents a higher heat sink for the gas phase; finally, the reduced evaporation rate for the $M L_{0}=0.5$ simulations renders the individual-drop 
a)

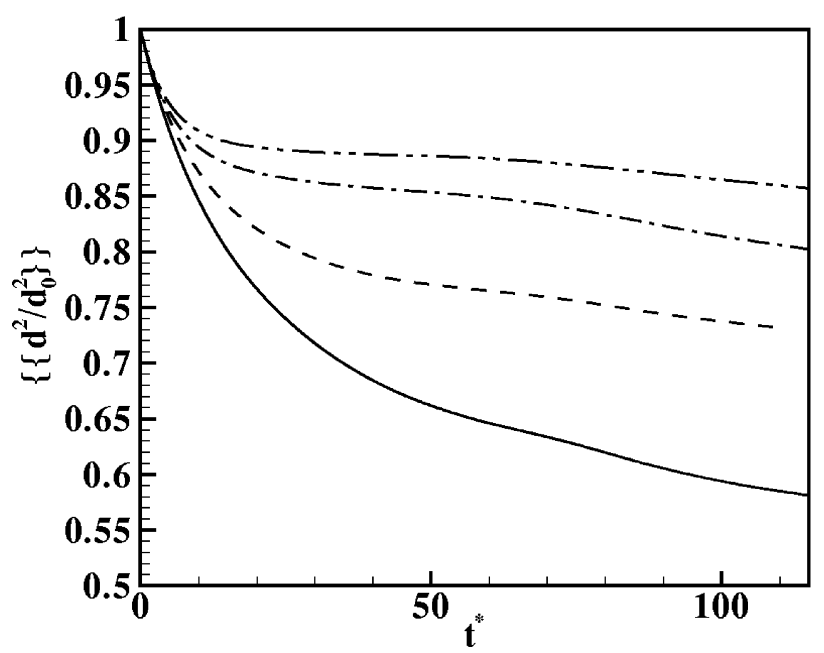

b)

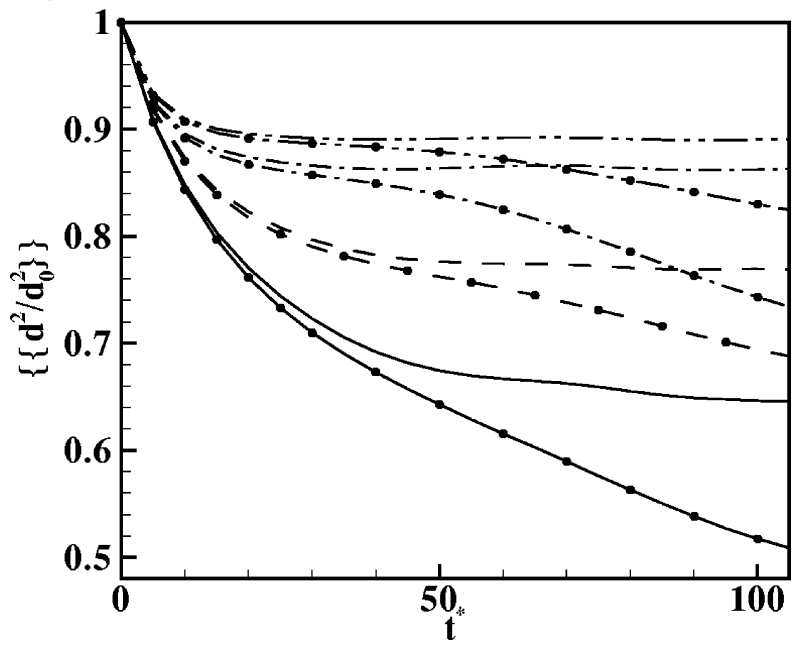

FIG. 11. Timewise evolution of the Lagrangian average residual droplet area for the SC and MC simulations. - - SC2; - - - MC2; -.- SC5; -.- MC5 and (a) for the entire domain, (b) separately for the lower stream (lines) defined as $x_{2} / \delta_{\omega, 0}<-7$ and the mixing layer (lines and symbols).

evaporation rate in each of those simulations more uniform in magnitude for reasons discussed in Sec. IV D 2. Comparisons of plots in Figs. 11(a) and 11(b) show that this general behavior is more typical of drops inside the layer. The drops in the lower stream reach an asymptotic size by the completion of the first pairing for $M L_{0}=0.2$ and by the end of rollup for $M L_{0}=0.5$, indicating cessation of evaporation due to saturation. The earlier saturation for $M L_{0}=0.5$ is due to the higher initial $\rho_{n}$ which induces a larger vapor accumulation in the lower stream.

Displayed in Fig. 12 is the PDF of St in the mixing layer for all simulations at transition. All four simulations were initiated with the same St PDF (Table II), and therefore evolving differences result from either the $M L_{0}$ value or the MC aspect; since $\mathrm{St}=\rho_{l} d^{2} \operatorname{Re}_{0} /\left(18 \rho \delta_{\omega, 0}^{2}\right) \sim d^{2} / \rho$ with all other quantities being fixed, Fig. 12 represents the $\rho$-modulated polydispersity evolution. The probability of being at the mean is practically independent of $M L_{0}$ for the $\mathrm{SC}$ simulations and slightly reduced for MC5 compared to MC2; the mean is reduced with decreasing $M L_{0}$ for each type of

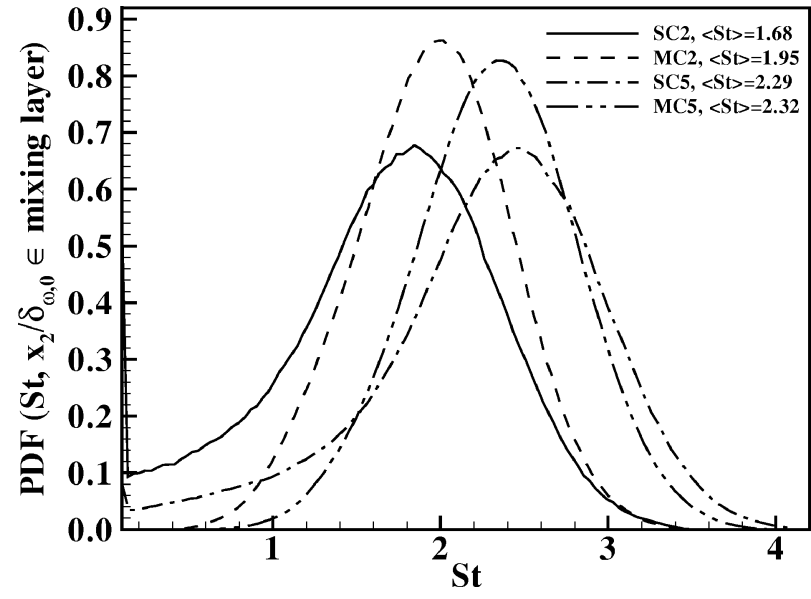

FIG. 12. Probabilty density functions of drop Stokes number inside the mixing layer (defined as $x_{2} / \delta_{\omega, 0} \geqslant-7$ ), each at the transitional time. simulation indicative of $\rho$-modulated enhanced evaporation. Independent of $M L_{0}$, the mean is larger and the probability of being at the mean is higher for MC simulations compared to their SC counterpart. This observation is consistent with the narrower St distribution for each MC simulation compared to the equivalent $\mathrm{SC}$ one, indicating a decreased $\rho$-modulated polydispersity resulting from the MC aspect. The larger $\rho$-modulated drop size and the $\rho$-modulated reduced polydispersity for $\mathrm{MC}$ cases are attributed to the combination of slower evaporation with condensation (discussed in Sec. IV D 2) occurring only in MC simulations.

\section{Drop composition and temperature}

In SC computations, the drop composition is inherently invariant. In MC simulations, condensation occurs as the result of the drops being transported in regions of the flow having different compositions. The condensation of these species is clearly evident in Figs. 13(a) and 13(c), where it is seen that although $\left\{\left\{\theta_{l}\right\}\right\}$ increases with time due to the evaporation of the lighter components, $\left\{\left\{\sigma_{l}\right\}\right\}$ exhibits a nonmonotonic behavior. Following an initial decrease due to the more volatile components leaving the liquid drop, $\left\{\left\{\sigma_{l}\right\}\right\}$ increases indicating that some mixture constituents that have already evaporated are now condensing back onto the drops; further variations in $\left\{\left\{\sigma_{l}\right\}\right\}$ indicate a slow, asymptotic evolution. This condensation process may also contribute to the larger $\rho$-modulated residual diameter of the MC-fuel drops observed in Figs. 11 and 12. The fate of the fuel composition for drops in different regions of the layer was examined by performing separate ensemble averages over drops in the lower stream (i.e., for $x_{2} / \delta_{\omega, 0}<-7$ ) and over drops in the mixing layer (defined here as the remaining of the domain). The results portrayed in Figs. 13(b) and 13(d) show that the lower stream drops reach relatively fast an asymptotic approximately fixed composition, whereas the mixing-layer drops change composition, as both $\left\{\left\{\theta_{l}\right\}\right\}$ and $\left\{\left\{\sigma_{l}\right\}\right\}$ con- 

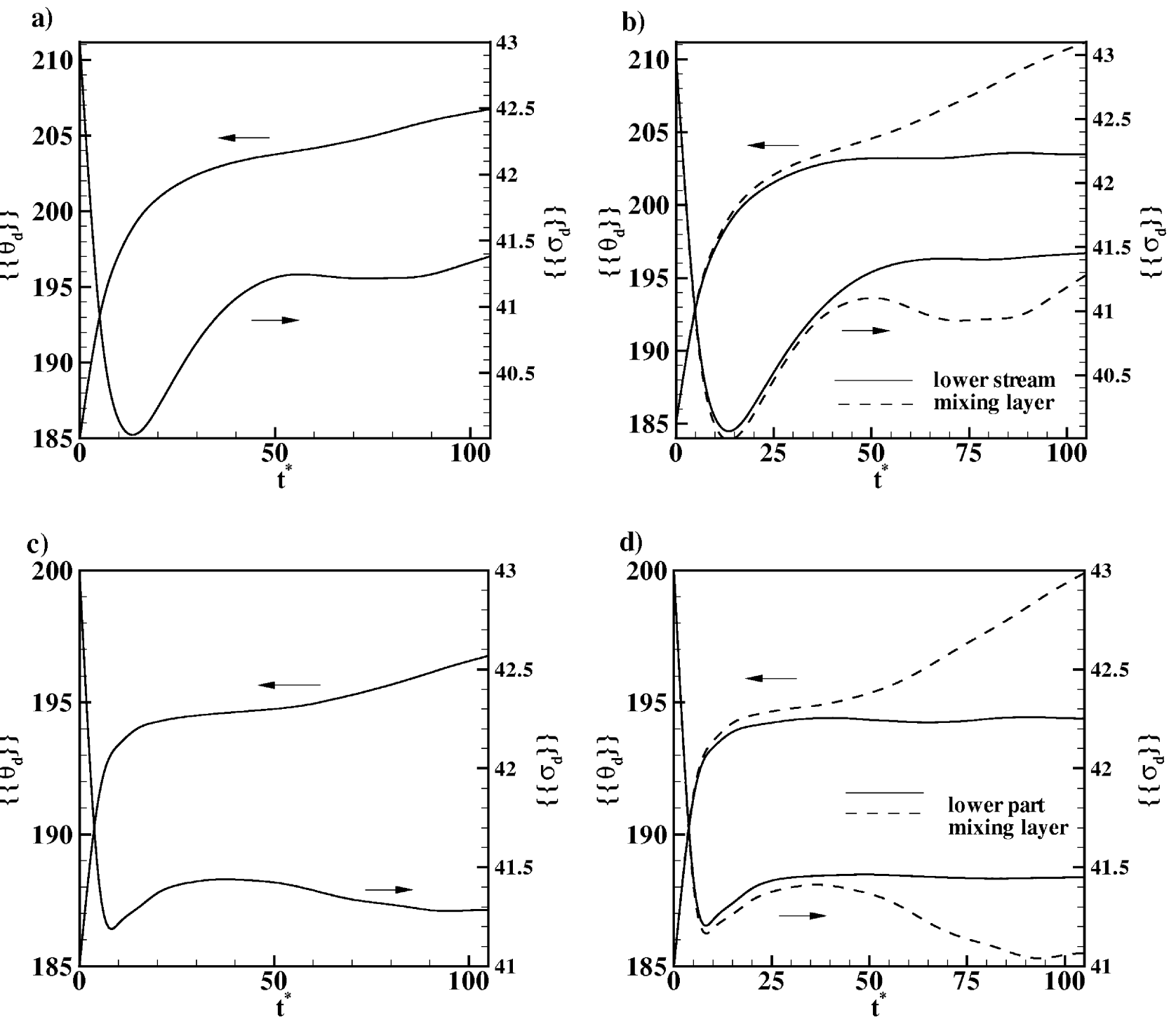

FIG. 13. Timewise evolution of the liquid composition in MC simulations: mean molar weight and variance in $\mathrm{kg} / \mathrm{kmole}$. (a) and (c) Lagrangian ensemble averages over the entire domain for MC2 and MC5, respectively. (b) and (d) Separate Lagrangian ensemble averages, for MC2 and MC5, respectively, over the lower stream, defined as $x_{2} / \delta_{\omega, 0}<-7$, and the mixing layer, defined as the remaining part of the domain.

tinue to vary. Whereas in the interior of the mixing layer $\left\{\left\{\theta_{l}\right\}\right\}$ increases with time, $\left\{\left\{\sigma_{l}\right\}\right\}$ exhibits a nonmonotonic behavior indicative of concomitant evaporation of some species and condensation of other species. Thus the MC-fuel drop composition is the result of two competing instantaneous processes: evaporation and condensation.

The condensation also explains the variation in the drop temperature depicted in Fig. 14 for the ensemble average drop temperature over the entire domain, over the lower stream and over the complementary part of the domain to the lower stream. Two distinct behaviors are encountered. In the lower stream and over the entire domain, $\left\{\left\{T_{d}\right\}\right\}$ exhibits an undulatory behavior with an amplitude that is less than $1 \%$ of the mean drop temperature, while in $x_{2} / \delta_{\omega, 0} \geqslant-7$ a more random variation is evident; the domain ensemble average is skewed by the lower stream where the majority of drops reside. The lack of direct association between $\left\{\left\{T_{d}\right\}\right\}$ and the flow is due to the drops being influenced by the flow through the history of their far-field conditions; since the slip velocity $\|\mathbf{u}-\mathbf{v}\|$ is generally non-null, the motion does not directly influence $\left\{\left\{T_{d}\right\}\right\}$.

In $x_{2} / \delta_{\omega, 0}<-7$ and over the entire domain, the SC-fuel $\left\{\left\{T_{d}\right\}\right\}$ initially decreases due to evaporation. The further increase in $\left\{\left\{T_{d}\right\}\right\}$ is due to drop heating as a result of heat transfer from the gas phase. This stage is followed by a reduction in $\left\{\left\{T_{d}\right\}\right\}$ corresponding to a stronger evaporation induced by the larger $\left\{\left\{T_{d}\right\}\right\}$. Therefore evaporation and heating combine to render $\left\{\left\{T_{d}\right\}\right\}$ mildly undulatory for the SC case. In contrast to the SC situation, the MC-fuel $\left\{\left\{T_{d}\right\}\right\}$ decreases for a much shorter time following the initial conditions, corresponding to the evaporation of the very light components. Following this stage, $\left\{\left\{T_{d}\right\}\right\}$ must increase before the heavier components leave the liquid, slowing down evaporation (see Fig. 11). When condensation is initiated (see Fig. 13), $\left\{\left\{T_{d}\right\}\right\}$ increases but this augmentation occurs at a smaller rate since the condensing vapor carries the higher temperature of the gas, thus facilitating evaporation of 
a)

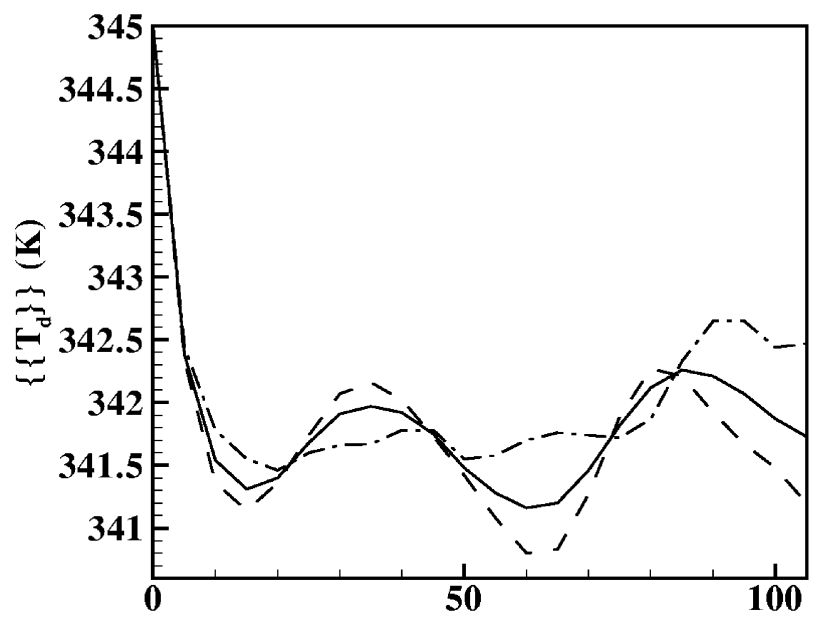

c)

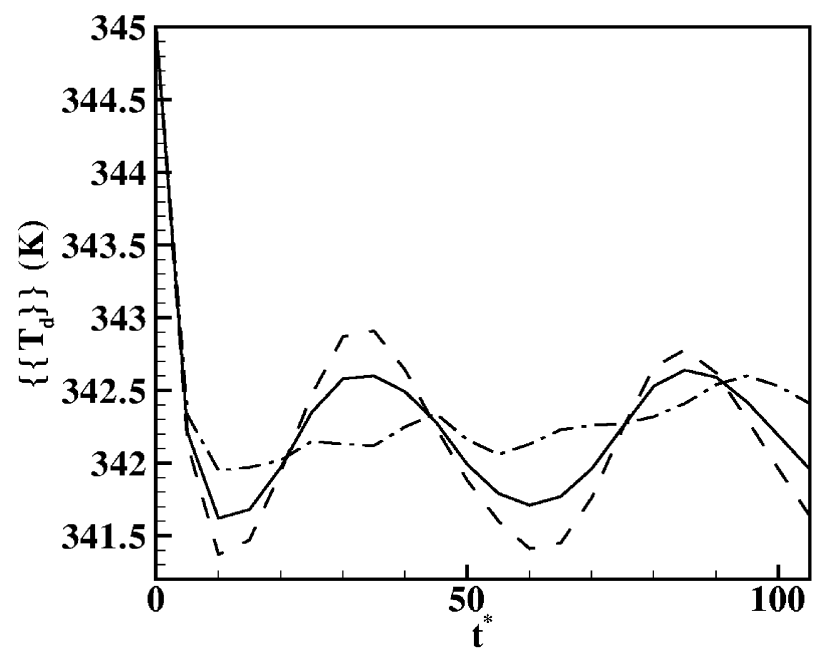

b)

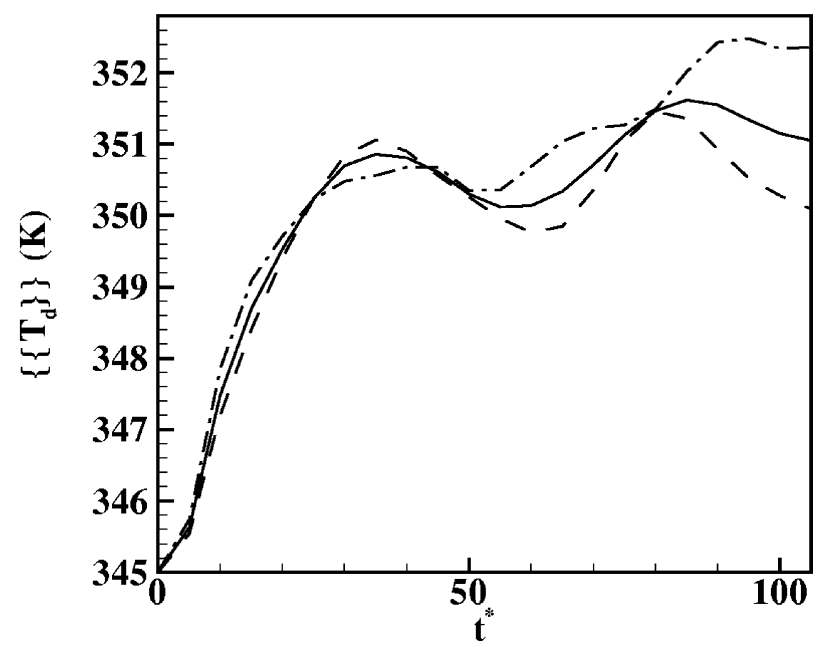

d)

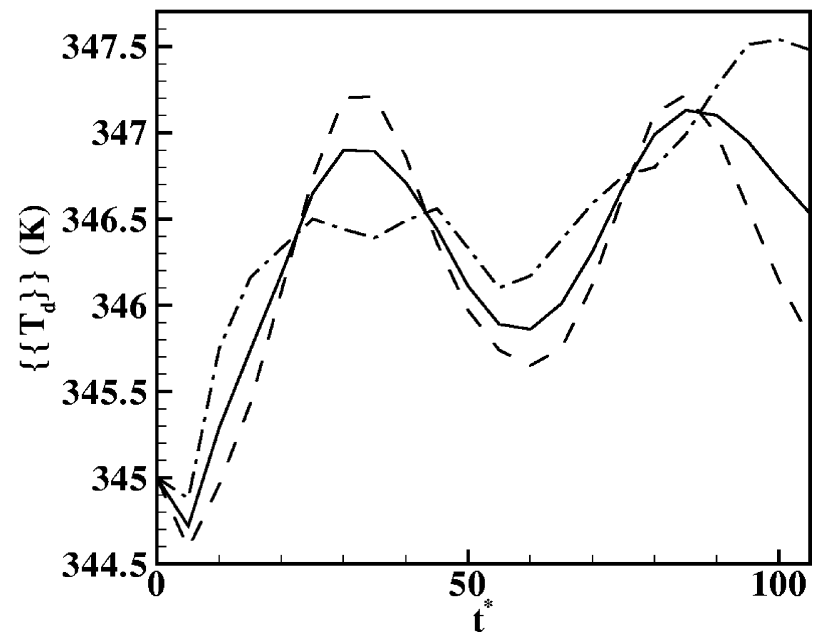

FIG. 14. Timewise evolution of the ensemble average drop temperature for the entire domain $(-)$, lower stream $(---)$, and the complementary part of the domain to the lower stream (-..-). (a) SC2, (b) MC2, (c) SC5, and (d) MC5.

other components in the drop. Eventually, the condensation rate decreases and $\left\{\left\{T_{d}\right\}\right\}$ is high enough to allow a stronger evaporation, explaining the further decrease in $\left\{\left\{T_{d}\right\}\right\}$. Following the evaporation of these intermediary molar weight species, $\left\{\left\{T_{d}\right\}\right\}$ must again increase before the higher molar weight components may evaporate. Thus the MC-fuel $\left\{\left\{T_{d}\right\}\right\}$ also has an undulatory behavior, but at a larger average than the SC-fuel drops. Figure 14 also shows that $\left\{\left\{T_{d}\right\}\right\}$ slightly increases with increasing $M L_{0}$ for SC simulations, but substantially decreases with increasing $M L_{0}$ for MC simulations, showing that this qualitative variation trend for MCfuel drops cannot be captured by the SC approximation. The more random evolution of $\left\{\left\{T_{d}\right\}\right\}$ in $x_{2} / \delta_{\omega, 0} \geqslant-7$ is the result of the drops interaction with the local turbulent flow.

\section{Vapor-fuel composition and gas temperature}

In SC simulations the fuel-vapor composition is inherently invariant. The impact of MC-fuel drop evaporation on the gas phase is illustrated in Fig. 15 showing the timewise evolution of $\left(x_{1}, x_{3}\right)$-plane averages for $\theta_{v}$ and $\sigma_{v}$ for MC2 [Figs. 15(a) and 15(b)] and MC5 [Figs. 15(c) and 15(d)]. Due to the early evaporation of the more volatile components, $\left\langle\theta_{v}\right\rangle$ rapidly increases in the lower, drop-laden stream. At later times, the drops in the lower stream continue evaporating and releasing species of increasing molar weight while the drops entrained into the layer begin releasing intermediary molar weight species, having already released their light molar weight components. As the drops are transported into the upper layer region, the site of the highest molar weight fractions in the gas phase changes accordingly, and the region occupied by the evaporated species widens. This is consistent with the location of the highest $\rho_{n}$ being in the upper stream (see Fig. 7) and with the cross-stream growth of the fuel-vapor layer (not shown). Finally, mixing induces the penetration of the intermediary molar-weight species into the lower part of the layer. With increasing $M L_{0}$, this penetration occurs earlier, however, the peak in $\left\langle\theta_{v}\right\rangle$ decreases, indicating a reduced species-stratification of the layer while evaporation in the lower stream ceases, with apparent satu- 
a)

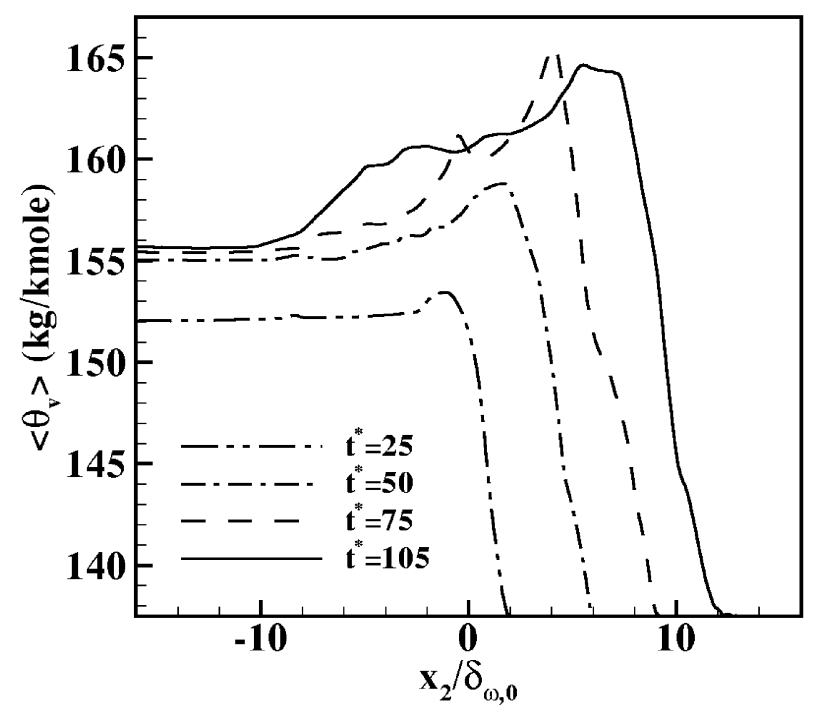

c)

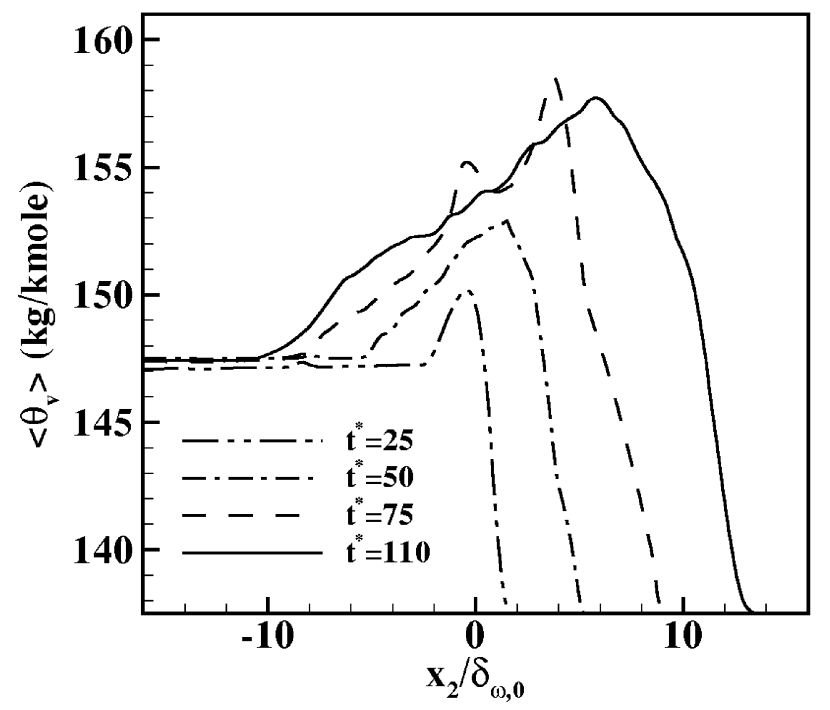

b)

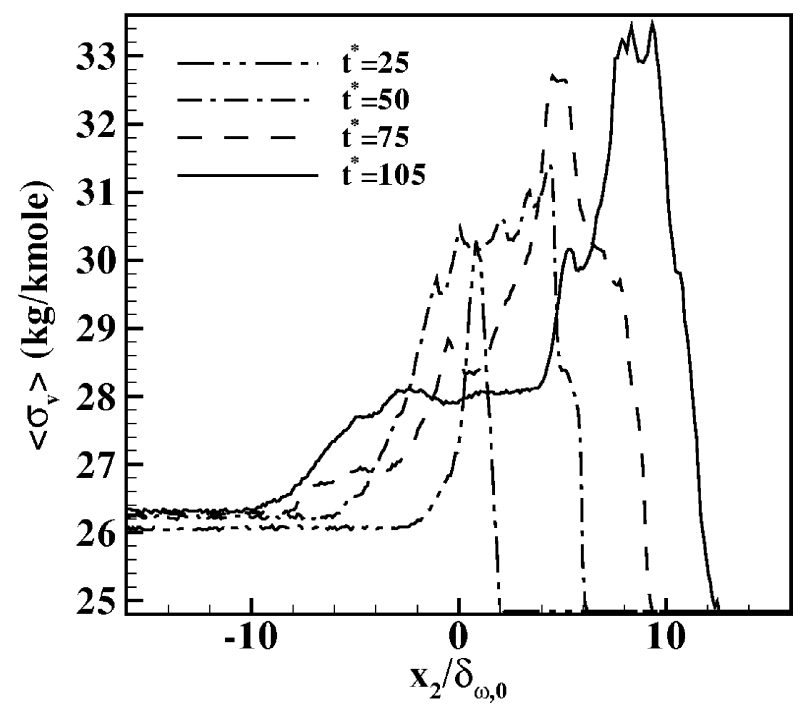

d)

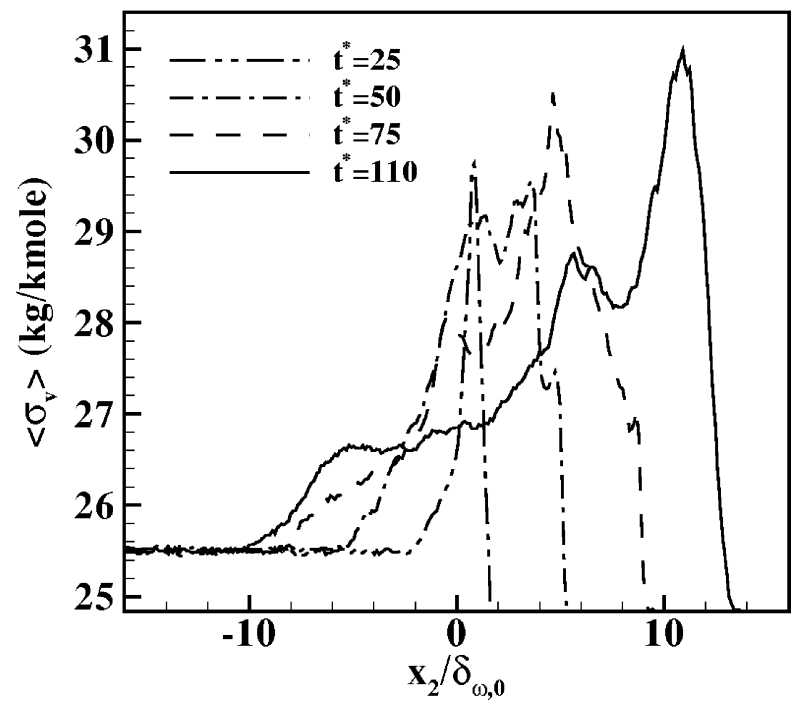

FIG. 15. Evolution of $\left(x_{1}, x_{3}\right)$-plane averages for (a) and (c) the vapor mean molar weight; (b) and (d) the vapor distribution-function variance. (a) and (b) for MC2; (c) and (d) for MC5. The times correspond to rollup, end of the first pairing, end of the second pairing, and transition.

ration. The indication of saturation is consistent with the information presented in Fig. 11 showing an asymptotic decrease in the drop size. It is now clear that in the upper part of the layer the drop size may evolve to be nearly constant, but this is the result of evaporation/condensation process, whereas in the lower stream the nearly constant drop size is an indication of saturation. This interpretation is supported by the plots in Figs. 15(b) and 15(d) illustrating $\left\langle\sigma_{v}\right\rangle$. Concomitant to the increase in $\left\langle\theta_{v}\right\rangle,\left\langle\sigma_{v}\right\rangle$ exhibits a similar augmentation. At earlier times, $\left\langle\sigma_{v}\right\rangle$ augmentation occurs in the region adjacent to the boundary between the two streams because this is where the drops encounter the highest gas temperature (heat transfer to the drops initially decreases the lower-stream gas temperature) and begin evaporating. The central peak in Figs. 15(b) and 15(d) at $t^{*}=25$ is equivalent to the corresponding peak in Figs. 15(a) and 15(c). The variance continues to display the largest augmentation in the region of strongest drop evaporation, as increasingly heavier components are released from the drops. At transition, the largest $\left\langle\sigma_{v}\right\rangle$ is in the upper part of the layer, indicating a more inhomogeneous molar weight region; the inhomogeneity decreases with increasing $M L_{0}$, emulating the $\left\langle\theta_{v}\right\rangle$ results. As one progresses towards the middle layer region, an abrupt reduction followed by a local peak is encountered, reminiscent of the nonuniformity in composition [e.g., Figs. 7(e) and 7(f)] around localities of high $\rho_{n}$ [e.g., Figs. 7(c) and $7(\mathrm{~d})]$. In the middle part of the layer, $\left\langle\sigma_{v}\right\rangle$ displays a plateau for $M L_{0}=0.2$, indicating a more uniform composition, while for $M L_{0}=0.5$ there is a gradual reduction from the upper to the lower part of the layer; this information reminds that of Figs. 7(e) and 7(f) which depicted the between-the-braid plane. At the lower extremity of the layer, another local peak is evident, but at a smaller $\left\langle\sigma_{v}\right\rangle$ than at the upper extremity of the layer, corresponding to the crossstream locations of large $\rho_{n}$ and larger $\left\langle\theta_{v}\right\rangle$ cross-stream variation, an example of which is clearly seen in Fig. 7. The 


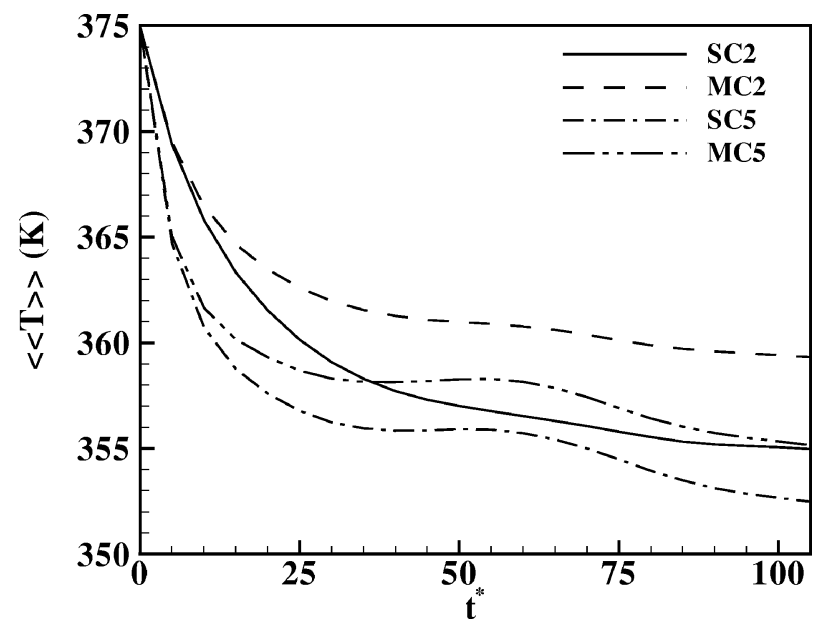

FIG. 16. Timewise evolution of the volume averaged gas temperature for the $\mathrm{SC}$ and $\mathrm{MC}$ simulations.

smallest $\left\langle\sigma_{v}\right\rangle$ is in the lower stream, indicating the most homogeneous region; while $\left\langle\sigma_{v}\right\rangle$ exhibits only a minimal timewise variation for $M L_{0}=0.2$, it displays no variation (on this $\left\langle\sigma_{v}\right\rangle$ scale) for $M L_{0}=0.5$, which completes the information indicative of saturation.

The volume-averaged gas temperature depicted in Fig. 16 initially decreases for both SC and MC cases. However, as the MC-fuel drop evaporation rate decreases, $\langle\langle T\rangle\rangle$ remains larger since the heat transfer is reduced. The $M L_{0}$ $=0.5$ simulations exhibit a plateau corresponding to the shallow part of the $\left\{\left\{T_{d}\right\}\right\}$ curves in Fig. 14 representing the duration between rollup and conclusion of the first pairing. The further reduction in $\langle\langle T\rangle\rangle$ mirrors the second augmentation in $\left\{\left\{T_{d}\right\}\right\}$ occurring after the first pairing.

\section{CONCLUSIONS}

A study of a multicomponent drop-laden threedimensional mixing layer has been conducted by adopting the continuous thermodynamics approach to mathematically describe the liquid composition in a probabilistic manner. Following previous continuous thermodynamics representations, the distribution of the chemical species in the fuel is described by a function of the molar weight. Initially, the layer is laden with drops in the lower stream and the drop temperature is lower than that of the carrier gas. Drop evaporation leads to a change in the gas-phase composition, which is also described in a probabilistic manner. A model for the MC-fuel drop-laden mixing layer has been developed by assuming the initial mathematical form of the distribution function and postulating that the same form is retained during the drop lifetime, but with evolving mean and variance as the drops evaporate. Therefore the physical complexity of the MC situation resulted in only two additional equations (for the mean and for the second moment) for each liquid and gas.

A test of the isolated drop model using this probabilistic approach was conducted and has shown that when the initial liquid-species distribution is narrow and has the same mean molar weight as the single-component fuel, the diameter predicted by the multicomponent model compares favorably with that of the single-component model. Further, isolated multicomponent-fuel drop calculations were conducted with diesel and gasoline fuels to elucidate their behavior when compared to that of $n$-decane.

The results from four mixing layer simulations were considered, where two simulations performed with dieselfuel drops were compared with two other simulations conducted with $n$-decane drops. ${ }^{13}$ Except for the liquid properties (density, composition, and thermophysical properties), all initial conditions based on the Mach, Reynolds, Prandtl, and Schmidt numbers, on the Stokes number probability distribution function, on the drop and gas temperature and on the gas composition were the same in all calculations. However, due to the different fuel density, the specification of the same initial Stokes number probability distribution function lead to smaller initial drop diameters and larger initial number of drops in the multicomponent case compared to the single-component equivalent.

The mixing layer simulations consisted of the perturbation-induced double pairing of four initial vortices to yield an ultimate vortex within which small scales proliferated, leading to transitional states. The global properties of the layers (momentum thickness, enstrophy, positive spanwise vorticity, and vorticity budgets) displayed modest sensitivity to the fuel composition and the layers attained transition at similar times. Visualizations of dynamic and thermodynamic variables at transition showed, however, that the details of the multicomponent-fuel layers differ from their single-component counterpart. Multicomponent-fuel drops evaporated slower due to the higher saturation pressure of the heavier species, leading to their interaction time with the flow being longer. This longer interaction time permitted the development of a more complex small-scale vorticity structure in the flow at transition, and the creation of regions of higher drop number density which also displayed more structure, particularly in high strain regions, than in the single-component fuel simulation. The last feature was the combined result of single-component fuel drops becoming evaporated, and thus being removed from the computation, and of the initially smaller number of drops, as explained above. In the single-component case, the molar weight of the evaporated fuel was inherently constant and its spatial distribution followed that of the evaporated fuel mass fraction. For the multicomponent drop case, evaporation leads to the mean molar weight in the liquid increasing and the variance initially decreasing. However, as drops were transported into regions of different gas compositions, condensation occurred, leading to an eventual increase of the liquid variance. This realistic condensation of some species coexisting with the evaporation of other species was captured with only two additional conservation equations compared to the singlecomponent fuel situation. The slower evaporation and the evaporation/condensation process were considered contributors to the reduced gas-density-modulated drop-size polydispersity in multicomponent simulations compared to their single-component counterpart. The species released from the 
drop contributed to increasing both the mean molar weight and the variance of the gas composition. Visualization of the mean molar weight spatial distribution in streamwise planes at transition revealed that the lighter components accumulated in the lower stream as they were released early during evaporation, before the drops were entrained in the layer. Intermediary molar weight species resided in the interior of the layer because they were released after the drops were entrained and therefore participated in the mixing process resulting from the double vortex pairing. The heavier components, which were released later in the drop lifetime, resided in regions of high drop-number density. Therefore, despite turbulence features, a segregation of the chemical species occurred based on the time of their release from the drops. It is this segregation, which is important in many processes, including combustion, that cannot be captured by the single-component fuel drop approximation.

Thus we conclude that drop-laden single-componentfuel experiments and simulations can reproduce the global aspects of equivalent multicomponent-fuel flows. However, the present results cast doubt on the ability of singlecomponent-fuel situations to represent important aspects of multicomponent-fuel flows; these include the increasing evaporation time which leads to the increased small-scale structure of the flow and particularly the species segregation which persists under transitional flow conditions.

\section{ACKNOWLEDGMENTS}

This research was performed at the Jet Propulsion Laboratory (JPL) of the California Institute of Technology, under the partial sponsorship of the Donors of The Petroleum Research Fund, administered by the American Chemical Society. Additional sponsorship was provided by U.S. Department of Energy (DOE), with Neil Rossmeissl (DOE Headquarters) and D. Hooker (DOE Golden Center) serving as contract monitors, under an agreement with the National Aeronautics and Space Administration. The authors wish to thank Dr. Kenneth Harstad of JPL for many helpful discussions on thermodynamics and Dr. Nora Okong'o of JPL for help with numerical and computational aspects. Computational resources were provided by the supercomputing facility at JPL.

\section{APPENDIX: CONSISTENT BOUNDARY CONDITIONS}

The characteristic boundary conditions (NSCBC) method in the context of the local one-dimensional inviscid LODI (as in Refs. 36 and 37) was implemented here to derive boundary conditions. This new derivation is necessary because the mean molar weight and the second moment become variables in the context of CT. The CT-modified Navier-Stokes system of equations can be written as

$$
\frac{\partial \widetilde{\mathbf{U}}}{\partial t}+\frac{\partial \mathbf{F}^{(k)}}{\partial x_{j}}+\widetilde{\mathbf{C}}^{(k)}=0,
$$

where the set of conservative variables is defined by

$$
\begin{aligned}
\widetilde{\mathbf{U}}= & \left(c, c m u_{j}, c e_{t}, c X_{v}, c X_{v} \theta_{v}, c X_{v} \psi_{v}\right), \\
& j \in[1,3],
\end{aligned}
$$

the flux vector of the conservative variables is the following:

$$
\begin{aligned}
\mathbf{F}^{(k)}= & {\left[c u_{j}, c u_{j} u_{k},\left(c e_{t}+p\right) u_{j}, c X_{v} u_{j}, c X_{v} \theta_{v} u_{j}, c X_{v} \psi_{v} u_{j}\right], } \\
& k \in[1,3],
\end{aligned}
$$

and $\widetilde{\mathbf{C}}^{(k)}$ includes all terms without any first derivative of $\widetilde{\mathbf{U}}$ along the $j$ direction,

$$
\begin{aligned}
\widetilde{\mathbf{C}}^{(k)=}= & -\frac{\partial}{\partial x_{j}} c \bar{D} \frac{\partial}{\partial x_{j}}\left(X_{v}\right)+\frac{1}{m_{g a}} \frac{\partial}{\partial x_{j}}\left(c \widetilde{D} \frac{\partial}{\partial x_{j}}\left(X_{v} \theta_{v}\right)\right)-S_{I-\text { mole }},-\frac{\partial \tau_{k j}}{\partial x_{j}}-S_{I I, k}, \\
& -\frac{\partial}{\partial x_{j}}\left[\lambda \frac{\partial T}{\partial x_{j}}+u_{i} \tau_{i j}-\sum_{\beta=[g a,[1, N]]} J_{D j \beta} h_{\beta}\right]-S_{I I I},-\frac{\partial}{\partial x_{j}}\left(c \bar{D} \frac{\partial X_{v}}{\partial x_{j}}\right)-S_{I-\text { mole }}, \\
& \left.-\frac{\partial}{\partial x_{j}}\left(c \widetilde{D} \frac{\partial}{\partial x_{j}}\left(X_{v} \theta_{v}\right)\right)-S_{I-\text { mass }},-\frac{\partial}{\partial x_{j}} c \hat{D} \frac{\partial}{\partial x_{j}}\left(X_{v} \psi_{v}\right)-S_{\Psi}\right\} .
\end{aligned}
$$

The system of equations is closed by using the perfect gas law for the gas mixture $p=c R_{u} T$.

In order to identify characteristic waves in the hyperbolic part of our CT-modified system of equations, we derive an equivalent form of the system using the following set of primitive variables: $\mathbf{U}=\left(c, u_{j}, p, X_{v}, \theta_{v}, \psi_{v}\right)$. If $\mathbf{P}$ is the ma- trix transformation from the conservative to the primitive variables,

$$
\mathbf{P}=\frac{\partial \widetilde{\mathbf{U}}}{\partial \mathbf{U}},
$$




$$
\mathbf{P}=\left(\begin{array}{cccccccc}
1 & 0 & 0 & 0 & 0 & 0 & 0 & 0 \\
m u_{1} & m c & 0 & 0 & 0 & c u_{1}\left(\theta_{v}-m_{g a}\right) & c u_{1} X_{v} & 0 \\
m u_{2} & 0 & m c & 0 & 0 & c u_{2}\left(\theta_{v}-m_{g a}\right) & c u_{2} X_{v} & 0 \\
m u_{3} & 0 & 0 & m c & 0 & c u_{3}\left(\theta_{v}-m_{g a}\right) & c u_{3} X_{v} & 0 \\
\mathbf{P}_{5,1} & c u_{1} & c u_{2} & c u_{3} & \frac{1}{\gamma-1} & \mathbf{P}_{5,6} & \mathbf{P}_{5,7} & 0 \\
X_{v} & 0 & 0 & 0 & 0 & c & 0 & 0 \\
X_{v} \theta_{v} & 0 & 0 & 0 & 0 & c \theta_{v} & c X_{v} & 0 \\
X_{v} \psi_{v} & 0 & 0 & 0 & 0 & c \psi_{v} & 0 & c X_{v}
\end{array}\right),
$$

where

$$
\begin{aligned}
& \mathbf{P}_{5,1}=\frac{u_{i} u_{i}}{2}+X_{v}\left(K \theta_{v}+K^{\prime}\right), \\
& \mathbf{P}_{5,6}=p\left(A_{c}+B_{c} \theta_{v}-\frac{C_{p g a}}{R_{u}}\right)+c\left(K \theta_{v}+K^{\prime}\right), \\
& \mathbf{P}_{5,7}=X_{v}\left(p B_{c}+c K\right) .
\end{aligned}
$$

The wave amplitudes corresponding to each eigenvalue $\lambda_{i}$ are

$$
\begin{aligned}
& \mathcal{L}_{1}=\left(u_{2}-c\right)\left[\frac{\partial p}{\partial y}-m c a \frac{\partial u_{2}}{\partial y}\right] \quad \text { for } \lambda_{1}=u_{2}-a \\
& \mathcal{L}_{2}=u_{2}\left[\frac{\partial p}{\partial y}-m a^{2} \frac{\partial c}{\partial y}\right] \text { for } \lambda_{2}=u_{2}, \\
& \mathcal{L}_{3}=u_{2} \frac{\partial u_{1}}{\partial y}, \quad \mathcal{L}_{4}=u_{2} \frac{\partial u_{3}}{\partial y} \text { for } \lambda_{3}=u_{2}, \quad \lambda_{4}=u_{2}, \\
& \mathcal{L}_{k}=u_{2} \frac{\partial \Omega_{k}}{\partial y} \text { for } \lambda_{k}=u_{2}, \quad k \in[5,7], \\
& \mathcal{L}_{8}=\left(u_{2}+c\right)\left[\frac{\partial p}{\partial y}+m c a \frac{\partial u_{2}}{\partial y}\right] \text { for } \lambda_{8}=u_{2}+a
\end{aligned}
$$

where $\Omega_{5}=X_{v}, \Omega_{6}=\theta_{v}, \Omega_{7}=\psi_{v}$. The cross stream boundaries are adiabatic slip walls, and therefore $\lambda(\partial T / \partial y)$ $+u_{2} \tau_{2 j}-\sum_{\beta=[g a,[1, N]]} J_{D 2 \beta} h_{\beta}=0$, reflecting the null heat flux condition through the wall. One inviscid condition, $u_{2}$ $=0$, must be satisfied at the slip wall, and the viscous relations are represented by null tangential stresses, $\tau_{12}=\tau_{23}$ $=0$. Since the normal velocity is null, the amplitudes $\mathcal{L}_{2}$ through $\mathcal{L}_{7}$ are null and $\mathcal{L}_{1}=\mathcal{L}_{8}$. The amplitude of the reflected wave $\mathcal{L}_{1}$ is thus set to $\mathcal{L}_{8}$, with $\mathcal{L}_{8}$ computed from the variables inside the domain.

${ }^{1}$ C. Epsey, J. E. Dec, T. A. Litzinger, and D. A. Santavicca, "Planar laser Rayleigh scattering for quantitative vapor-fuel imaging in a diesel jet," Combust. Flame 109, 65 (1997).

${ }^{2}$ J. D, Naber and R. D. Reitz, "Modeling engine spray/wall impingement," SAE Paper 880107, 1988.

${ }^{3}$ A. M. Lippert, D. W. Stanton, C. J. Rutland, R. D. Reitz, and W. H. L. Hallett, "Investigating the effect of spray targeting and impingement on diesel engine cold start," SAE Trans. 109, 250 (2000).

${ }^{4}$ G.-S. Zhu and R. D. Reitz, "Engine fuel droplet high-pressure vaporiza- tion modeling," ASME J. Eng. Gas Turbines Power 123, 412 (2001).

${ }^{5}$ A. M. Lippert, D. W. Stanton, C. J. Rutland, W. L. H. Hallett, and R. D. Reitz, "Multi-dimensional simulation of diesel engine cold-start with advanced physical submodels," Int. Journal of Engine Research 1, 1 (2000).

${ }^{6}$ M. Boivin, S. Simonin, and K. D. Squires, "Direct numerical simulation of turbulence modulation by particles in isotropic turbulence," J. Fluid Mech. 375, 235 (1998).

${ }^{7}$ F. Mashayek and F. A. Jaberi, "Particle dispersion in forced isotropic low-Mach-number turbulence," Int. J. Heat Mass Transfer 42, 2823 (1999).

${ }^{8}$ F. Mashayek, "Direct numerical simulations of evaporating droplet dispersion in forced low Mach number turbulence," Int. J. Heat Mass Transfer 41, 2601 (1998).

${ }^{9}$ J. Réveillon and L. Vervisch, "Spray vaporization in nonpremixed turbulent combustion modeling: A single drop model," Combust. Flame 121, 75 (2000).

${ }^{10} \mathrm{~F}$. Mashayek, "Droplet-turbulence interactions in low-Mach-number homogeneous shear two-phase flows," J. Fluid Mech. 367, 163 (1998).

${ }^{11}$ R. S. Miller and J. Bellan, "Direct numerical simulation of a confined three-dimensional gas mixing layer with one evaporating hydrocarbondroplet laden stream," J. Fluid Mech. 384, 293 (1999).

${ }^{12}$ R. S. Miller and J. Bellan, "Direct numerical simulation and subgrid analysis of a transitional droplet laden mixing layer," Phys. Fluids 12, 650 (2000).

${ }^{13} \mathrm{~N}$. Okong'o and J. Bellan, "Consistent large eddy simulation of a temporal mixing layer laden with evaporating drops. Part I: Direct numerical simulation, formulation and a priori analysis," J. Fluid Mech. 499, 1 (2004).

${ }^{14} \mathrm{~K}$. Harstad and J. Bellan, "A Model of the evaporation of binary-fuel clusters of drops," Atomization Sprays 1, 367 (1991).

${ }^{15}$ C. M. Megaridis and W. A. Sirignano, "Numerical modeling of a vaporizing multicomponent droplet," Proc. Comb. Inst. 23, 1413 (1990).

${ }^{16}$ C. K. Law and H. K. Law, "A $d^{2}$-law for multicomponent droplets vaporization and combustion," AIAA J. 20, 522 (1982).

${ }^{17}$ B. Gal-Or, H. T. Cullinan, Jr., and R. Galli, "New thermodynamictransport theory for systems with continuous component density distributions," Chem. Eng. Sci. 30, 1085 (1975).

${ }^{18}$ R. L. Cotterman, R. Bender, and J. M. Prausnitz, "Phase equilibria for mixtures containing very many components. Development and application of continuous thermodynamics for chemical process design," Ind. Eng. Chem. Process Des. Dev. 24, 194 (1985).

${ }^{19}$ J. R. Bowman and W. C. Edmister, "Flash distillation of an indefinite number of components," Ind. Eng. Chem. 43, 2625 (1951).

${ }^{20}$ W. C. Edmister and J. R. Bowman, "Equilibrium conditions of flash vaporization of petroleum fractions," Chem. Eng. Prog., Symp. Ser. 48, 46 (1952).

${ }^{21}$ J. G. Briano and E. D. Glandt, "Molecular thermodynamics of continuous mixtures," Fluid Phase Equilib. 14, 91 (1983).

${ }^{22}$ M. T. Rätzsch and H. Kehlen, "Continuous thermodynamics model of complex mixtures," Fluid Phase Equilib. 14, 225 (1983).

${ }^{23}$ J. Tamim and W. L. H. Hallett, "A continuous thermodynamics model for multicomponent droplet vaporization," Chem. Eng. Sci. 50, 2933 (1995).

${ }^{24} \mathrm{C}$. H. Whitson, "Characterizing hydrocarbon plus fractions," SPEJ 23, 683 (1983).

${ }^{25}$ G. F. Chou and J. M. Prausnitz, "Adiabatic flash calculations for continu- 
ous or semicontinuous mixtures using an equation of state," Fluid Phase Equilib. 30, 75 (1986).

${ }^{26}$ W. L. H. Hallett, "A simple model for the vaporization of droplets with large numbers of components," Combust. Flame 121, 334 (2000).

${ }^{27}$ A. M. Lippert and R. D. Reitz, "Modeling of multicomponent fuels using continuous distributions with application to droplet evaporation and sprays," SAE Paper 97FL-468, 1997.

${ }^{28}$ F. A. Williams, Combustion Theory (Addison-Wesley, Reading, MA, 1965).

${ }^{29}$ A. Makino and C. K. Law, "On the controlling parameter in the gasification behavior of multicomponent droplets," Combust. Flame 73, 331 (1988).

${ }^{30}$ R. S. Miller, K. Harstad, and J. Bellan, "Evaluation of equilibrium and non-equilibrium evaporation models for many-droplet gas-liquid flow simulations,' Int. J. Multiphase Flow 24, 1025 (1998).

${ }^{31}$ G. K. Batchelor, An Introduction to Fluid Thermodynamics (Cambridge University Press, Cambridge, England, 1967).

${ }^{32}$ S. C. Wong and A. R. Lin, "Internal temperature distribution of droplets vaporizing in high temperature convective flows," J. Fluid Mech. 237, 671 (1992).
${ }^{33}$ X. Q. Chen and J. C. F. Pereira, "Computation of turbulent evaporating sprays with well-specified measurements: A sensitivity study on droplet properties,” Int. J. Heat Mass Transfer 39, 441 (1996).

${ }^{34}$ B. Abramzon and W. A. Sirignano, "Droplet vaporization model for spray combustion calculations," Int. J. Heat Mass Transfer 32, 1605 (1989).

${ }^{35}$ R. D. Moser and M. M. Rogers, "Mixing transition and the cascade to small scales in a plane mixing layer," Phys. Fluids A 3, 1128 (1991).

${ }^{36}$ T. J. Poinsot and S. K. Lele, "Boundary conditions for direct simulations of compressible viscous flows," J. Comput. Phys. 101, 104 (1992).

${ }^{37}$ M. Baum, T. J. Poinsot, and S. K. Lele, “Accurate boundary conditions for multicomponent reactive flows," J. Comput. Phys. 116, 247 (1994).

${ }^{38}$ K. D. Squires and J. K. Eaton, "Preferential concentration of particles by turbulence," Phys. Fluids A 3, 1169 (1991).

${ }^{39}$ C. T. Crowe, J. N. Chung, and T. R. Troutt, "Particle mixing in free shear flows," Prog. Energy Combust. Sci. 14, 171 (1988).

${ }^{40}$ G. Continillo and W. A. Sirignano, "Numerical study of multicomponent fuel spray flame propagation in a spherical closed volume," Proc. Comb. Inst. 22, 1941 (1988). 